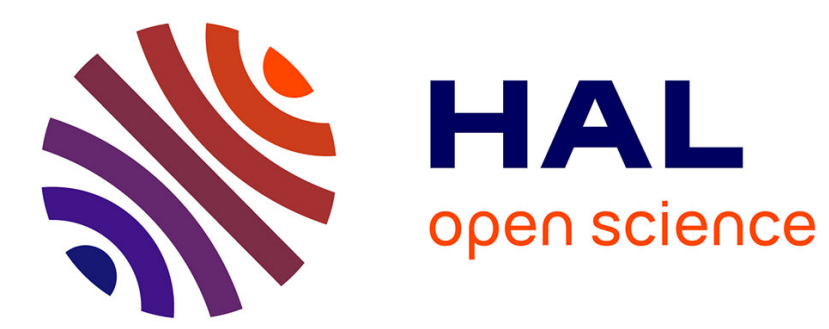

\title{
Slices for biparabolics of index one
}

Anthony Joseph, Florence Fauquant-Millet

\section{To cite this version:}

Anthony Joseph, Florence Fauquant-Millet. Slices for biparabolics of index one. Transformation Groups, 2011, 16 (4), pp.1081-1113. 10.1007/s00031-011-9158-1 . hal-00863222

\section{HAL Id: hal-00863222 \\ https://hal.science/hal-00863222}

Submitted on 19 Sep 2013

HAL is a multi-disciplinary open access archive for the deposit and dissemination of scientific research documents, whether they are published or not. The documents may come from teaching and research institutions in France or abroad, or from public or private research centers.
L'archive ouverte pluridisciplinaire HAL, est destinée au dépôt et à la diffusion de documents scientifiques de niveau recherche, publiés ou non, émanant des établissements d'enseignement et de recherche français ou étrangers, des laboratoires publics ou privés. 


\title{
SLICES FOR BIPARABOLICS OF INDEX $1^{1}$
}

\author{
ANTHONY Joseph \\ Donald Frey Professional Chair \\ Department of Mathematics \\ Weizmann Institute of Science \\ 2 Herzl Street \\ Rehovot, 76100, Israel \\ anthony.joseph@weizmann.ac.il
}

\author{
Florence Fauquant-Millet \\ Université de Lyon, F-42023 Saint-Etienne, France \\ Laboratoire de Mathématiques de l'Université de Saint-Etienne \\ Faculté des Sciences et Techniques \\ 23, rue du Docteur Paul Michelon \\ F-42023 Saint-Etienne Cédex 02 France \\ florence.millet@univ-st-etienne.fr
}

Key Words: Invariants, Slices, Nil-cones.

AMS Classification: 17B35

\begin{abstract}
Let $\mathfrak{a}$ be an algebraic Lie subalgebra of a simple Lie algebra $\mathfrak{g}$ with index $\mathfrak{a} \leq$ rank $\mathfrak{g}$. Let $Y(\mathfrak{a})$ denote the algebra of $\mathfrak{a}$ invariant polynomial functions on $\mathfrak{a}^{*}$. An algebraic slice for $\mathfrak{a}$ is an affine subspace $\eta+V$ with $\eta \in \mathfrak{a}^{*}$ and $V \subset \mathfrak{a}^{*}$ a subspace of dimension index $\mathfrak{a}$ such that restriction of function induces an isomorphism of $Y(\mathfrak{a})$ onto the algebra $R[\eta+V]$ of regular functions on $\eta+V$.

Slices have been obtained in a number of cases through the construction of an adapted pair $(h, \eta)$ in which $h \in \mathfrak{a}$ is ad-semisimple, $\eta$ is a regular element of $\mathfrak{a}$ which is an eigenvector for $h$ of eigenvalue minus one and $V$ is an $h$ stable complement to $(\operatorname{ad} \mathfrak{a}) \eta$ in $\mathfrak{a}^{*}$. The classical case is for $\mathfrak{g}$ semisimple [15, [16]. Yet rather recently many other cases have been provided. For example if $\mathfrak{g}$ is of type $A$ and $\mathfrak{a}$ is a "truncated biparabolic" [11] or a centralizer [12]. In some of these cases (particular when the biparabolic is a Borel subalgebra) it was found [12, [13], that $\eta$ could be taken to be the restriction of a regular nilpotent element in $\mathfrak{g}$. Moreover this calculation suggested [12] how to construct slices outside type $A$ when no adapted pair exists.

This article makes a first step in taking these ideas further. Specifically let $\mathfrak{a}$ be a truncated biparabolic of index one. (This only arises if $\mathfrak{g}$ is of type $A$ and $\mathfrak{a}$ is the derived algebra of a parabolic subalgebra whose Levi factor has just two blocks whose sizes are coprime.) In this case it is shown that the second member of an adapted pair $(h, \eta)$ for $\mathfrak{a}$ is the restriction of a particularly carefully chosen regular nilpotent element of $\mathfrak{g}$.

A by-product of the present analysis is the construction of an invariant associated to a pair of coprime integers.
\end{abstract}

Date: September 18, 2013.

${ }^{1}$ Work supported in part by Israel Science Foundation Grant, no. 710724. 


\section{INTRODUCTION}

Unless mentioned to the contrary the base field $\mathbb{K}$ is assumed algebraically closed of characteristic zero.

1.1. Invariants. Let $\mathfrak{a}$ be a finite dimensional Lie algebra, $S(\mathfrak{a})$ its symmetric algebra and $K(\mathfrak{a})$ the field of fractions of $S(\mathfrak{a})$. If $A$ is algebra in which $\mathfrak{a}$ acts by derivations, set $A^{\mathfrak{a}}=\{a \in A \mid x a=0, \forall x \in \mathfrak{a}\}$. It is a subalgebra of $A$.

Given $\xi \in \mathfrak{a}^{*}$, set $\mathfrak{a}^{\xi}=\{a \in \mathfrak{a} \mid a \xi=0\}$, that is the stabilizer of $\xi$ under co-adjoint action. It is a Lie subalgebra of $\mathfrak{a}$.

Define index $\mathfrak{a}:=\min _{\xi \in \mathfrak{a}^{*}} \operatorname{dim} \mathfrak{a}^{\xi}$. Set $\mathfrak{a}_{r e g}^{*}=\left\{\xi \in \mathfrak{a}^{*} \mid \operatorname{dim} \mathfrak{a}^{\xi}=\right.$ index $\left.\mathfrak{a}\right\}$, called the set of regular elements of $\mathfrak{a}^{*}$.

A problem of Dixmier [3, Problem 4] suggests that $C(\mathfrak{a}):=K(\mathfrak{a})^{\mathfrak{a}}$, is always a pure transcendental extension of $\mathbb{K}$.

One may further ask under what conditions is $Y(\mathfrak{a}):=S(\mathfrak{a})^{\mathfrak{a}}$ a polynomial algebra.

1.2. Slices. In [13, Sect. 7] we focused some attention on refinements of these questions. Here it is convenient to assume that $S(\mathfrak{a})$ admits no proper semi-invariants. In this case $C(\mathfrak{a})$ is just the field of fractions of $Y(\mathfrak{a})$. Moreover under this hypothesis, Ooms and Van den Bergh [17, Prop. 4.1] have shown that the growth rate (that is Gelfand-Kirillov dimension) of $Y(\mathfrak{a})$ takes its maximum possible value, namely index $\mathfrak{a}$.

Under the above hypothesis define a rational slice to be an affine translate $\eta+V \subset \mathfrak{a}^{*}$ of a vector subspace of $V$ of $\mathfrak{a}^{*}$ such that the restriction of functions gives on injection $\theta$ of $Y(\mathfrak{a})$ into the algebra of regular functions $R[\eta+V]$ on $\eta+V$ and induces an isomorphism of fields of fractions. Observe that $R[\eta+V]$ identifies with $S\left(V^{*}\right)$ and then comparison of transcendence degrees implies that $\operatorname{dim} V=$ index $\mathfrak{a}$. We call $\eta$ the base point of the slice $\eta+V$.

We suggested that a rational slice always exists [13, 7.11].

Define an algebraic slice to be a rational slice for which $\theta$ is an isomorphism. Obviously this implies that $Y(\mathfrak{a})$ is a polynomial algebra; but we found an example ([13, 11.4, Example 2]) for which the converse is false.

In view of this counter-example it would seem appropriate to suggest that if $Y(\mathfrak{a})$ is polynomial then there exists an affine subspace $\eta+V \subset \mathfrak{a}^{*}$ such that restriction of functions gives an embedding $Y(\mathfrak{a}) \hookrightarrow R[\eta+V] \stackrel{\sim}{\rightarrow} S\left(V^{*}\right)$ whose image takes the form $S\left(V^{*}\right)^{G}$ for some finite (pseudo-reflection) group $G$ acting linearly on $V^{*}$.

Finally we remark that the notions of a rational or algebraic slice were given ([13, Sect. 7] natural geometric interpretations in the case when $A$ is a connected algebraic group with Lie algebra $\mathfrak{a}$, that is when $\mathfrak{a}$ is algebraic. In particular $A(\eta+V)$ must be dense [13, 7.9] (but not necessarily open [13, 11.4, Example 3]) in $\mathfrak{a}^{*}$. Thus $\eta+V$ must meet most regular orbits (defined as those of codimension equal to index $\mathfrak{a}$ ). However even in the case of an algebraic slice not every regular orbit need pass [8, 8.12(ii)] through $\eta+V$, nor need every orbit meeting $\eta+V$ be regular [13, 11.4, Example 3]. In particular the base point $\eta$ need not be regular. 
1.3. Adapted Pairs. An adapted pair $(h, \eta)$ for a finite dimensional Lie algebra consists of a regular element $\eta \in \mathfrak{a}^{*}$ and an element $h \in \mathfrak{a}$ such $h \eta=-\eta$ with respect to co-adjoint action. Such pairs are rather hard to find, it being particularly difficult to check regularity.

Assume that $\mathfrak{a}$ is an algebraic Lie algebra. Then in the above we may use Jordan decomposition to show that the (adjoint) action of $h$ on $\mathfrak{a}$ can be taken to be reductive

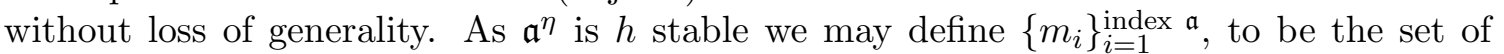
eigenvalues (counted with multiplicities) of $-h$ acting on $\mathfrak{a}^{\eta}$. These can be rather arbitrary and may depend on the choice of the adapted pair [14, 8.3]. However suppose that $S(\mathfrak{a})$ admits no proper semi-invariants and that $Y(\mathfrak{a})$ is polynomial. Then by [14, Cor. 2.3] the degrees of the homogeneous generators of $Y(\mathfrak{a})$ are the $m_{i}+1: i=1,2, \ldots$, index $\mathfrak{a}$, and moreover $\eta+V$ is an algebraic slice for any $h$ stable complement $V$ to $\mathfrak{a} \eta$ in $\mathfrak{a}^{*}$. (This is actually proved under a slightly weaker hypothesis which allows $\mathfrak{a}$ to be the centralizer $\mathfrak{g}^{x}$ in a semisimple Lie algebra $\mathfrak{g}$.) Thus the $\left\{m_{i}\right\}$ generalize the so-called "exponents" defined classically for $\mathfrak{a}$ semisimple.

In the above situation every element of $\eta+V$ is regular (see for example [13, 7.8]) by a standard deformation argument.

1.4. The Nilpotent Cone. One would like to have a systematic way to construct algebraic slices. In this we make the rather bold suggestion below. It should be regarded more as a signpost rather than a serious conjecture.

Suppose that $\mathfrak{a}$ is an algebraic subalgebra of a semisimple Lie algebra $\mathfrak{g}$. Let $G$ be the adjoint group of $\mathfrak{g}$ and $A$ the unique closed subgroup whose Lie algebra is $\mathfrak{a}$.

Assume that index $\mathfrak{a} \leq \operatorname{rank} \mathfrak{g}$. This is the case if $\mathfrak{a}$ is a biparabolic subalgebra [5], 7] or a centralizer (via the now known truth of the Elashvili conjecture [2], [6], [21]).

Further assume that $\mathfrak{g}$ admits a Chevalley antiautomorphism $\kappa$ such that $\mathfrak{a}$ and $\kappa(\mathfrak{a})$ are non-degenerately paired through the Killing form $K$ on $\mathfrak{g}$. This is clearly the case for (truncated) biparabolics. It is well-known for a centralizer - see [14, 4.5,4.6] for details and references. It is less clear that this condition is really necessary. We use it mainly for convenience.

Under the above hypothesis we may and will identify $\mathfrak{a}^{*}$ with the subalgebra $\kappa(\mathfrak{a})$ of $\mathfrak{g}$.

Let $\mathfrak{k}$ be the kernel of the restriction map $\mathfrak{g}^{*} \rightarrow \mathfrak{a}^{*}$. Identifying $\mathfrak{g}^{*}$ with $\mathfrak{g}$ through the Killing form we may view $\mathfrak{k}$ as a subspace of $\mathfrak{g}$.

Obviously $\mathfrak{k}$ is $A$ stable. In view of the above identifications we may write $A(\xi+\mathfrak{k})=$ $A \xi+\mathfrak{k}$, for all $\xi \in \mathfrak{a}^{*}$. In particular if $\xi \in \mathfrak{a}_{\text {reg }}^{*}$, then $\operatorname{codim} A(\xi+\mathfrak{k}) \leq \operatorname{rank} \mathfrak{g}$.

Let $\mathscr{N}(\mathfrak{g})$ denote the cone of ad-nilpotent elements of $\mathfrak{g}$. As is well-known, codim $\mathscr{N}(\mathfrak{g})=$ rank $\mathfrak{g}$. Moreover $\mathscr{N}(\mathfrak{g})$ is irreducible and admits only finitely many $G$ orbits. In particular $\mathscr{N}(\mathfrak{g})_{\text {reg }}$ consists of a dense open orbit.

Now let $(h, \eta)$ be an adapted pair for $\mathfrak{a}$. The relation $h \eta=-\eta$, forces $\eta \in \mathscr{N}(\mathfrak{g})$. However it is almost never the case that $\eta \in \mathscr{N}(\mathfrak{g})_{\text {reg. }}$ (For example, in [11, Sect. 10] a detailed study of the nilpotent orbit to which $\eta$ belongs was made in the case of an adapted pair $(h, \eta)$ of a truncated biparabolic in type $A$.)

In view of the above codimensionality estimates we propose the

Suggestion. Suppose $(h, \eta)$ is an adapted pair for $\mathfrak{a}$. Then $\eta+\mathfrak{k} \cap \mathscr{N}(\mathfrak{g})_{\text {reg }}$ is non-empty. 
Remarks. This just means that there is some pre-image of $\eta \in \mathfrak{g}^{*}$ lying in $\mathscr{N}(\mathfrak{g})_{\text {reg. }}$ It could be proved by showing that the codimension of $\mathscr{N}(\mathfrak{g}) \cap A(\eta+\mathfrak{k})$ in $\mathfrak{g}^{*}$ equals rank $\mathfrak{g}$, though this is likely to be rather difficult if even true. It suggests that one should construct $\eta$ as the restriction of some element of $\mathscr{N}(\mathfrak{g})_{\text {reg }}$. The fact that $\eta$ itself does not belong to $\mathscr{N}(\mathfrak{g})_{\text {reg }}$ is just a consequence of having made a particular choice of its pre-image in $\mathfrak{g}$. However it is this choice which allows one to guess $\eta$, itself a rather hard task as explained in [11, 1.3]. A main point that lies behind our suggestion is that there may be a more "canonical" choice which leads to a slice making sense for a biparabolic or centralizer of an arbitrary semisimple Lie algebra. For the moment it is not too clear if this can be divined. Hopefully the present article will provide a clue. Here we should also stress that adapted pairs are far from unique even up to the obvious conjugation [11, 1.4]. Our suggestion is also partly motivated by Question (5) of [11, Sect. 11].

1.5. Let us recall some cases in which the above suggestion has a positive answer.

Choose a Cartan subalgebra $\mathfrak{h}$ of $\mathfrak{g}$ and let $\Delta \subset \mathfrak{h}^{*}$ denote the set of non-zero roots of $\mathfrak{g}$ with respect to $\mathfrak{h}$. For each $\alpha \in \Delta$ let $x_{\alpha}$ be a non-zero vector in $\mathfrak{g}$ of weight $\alpha$.

let $\pi \subset \Delta$ be a choice of simple roots and set $\Delta^{+}=\Delta \cap \mathbb{N} \pi, \mathfrak{n}=\sum_{\alpha \in \Delta^{+}} \mathbb{K} x_{\alpha}$ and $\mathfrak{b}=\mathfrak{h}+\mathfrak{n}$, which is a Borel subalgebra. Let $N, H, B$ be the corresponding closed subgroups of $G$.

First assume that $\mathfrak{a}$ is a centralizer, that is of the form $\mathfrak{g}^{x}$. (Here we can assume $x$ nilpotent without loss of generality and we shall always do this.)

Suppose $\xi$ is a regular element of $\left(\mathfrak{g}^{x}\right)^{*}$, for example coming from the second factor in an adapted pair. Then under the identifications made in 1.4, it follows from [12, Lemma 2.2] (which was inspired by the proof of the Vinberg inequality) and the truth of the Elashvili conjecture that $x+t \xi$ is a regular element of $\mathfrak{g}^{*}$ for all $t$ belonging to a cofinite subset $\Omega \subset \mathbb{K}$. However in general $x+t \xi$ will not be nilpotent. Rather for $\xi$ in general position $x+t \xi: t \in \Omega$ will be semisimple [12, 5.5].

Conversely if $\mathfrak{g}$ is of type $A$, then we may take $x$ in Jordan form (defined by an ordered partition $\mathbf{x}$ of $n$ ) and then "complete" it to a standard regular nilpotent element. More precisely up to conjugation we can write $\sum_{\alpha \in \pi^{\prime}} x_{\alpha}$, with $\pi^{\prime} \subset \pi$ corresponding to $\mathbf{x}$. Set $y^{\prime}=\sum_{\alpha \in \pi \backslash \pi^{\prime}} x_{\alpha}$. Then $x+y^{\prime}=\sum_{\alpha \in \pi} x_{\alpha}$, which is the standard presentation of a regular nilpotent element.

Now consider $y^{\prime}$ as an element of $\left(\mathfrak{g}^{x}\right)^{*}$ through the Killing form $K$. (With respect to what we said in 1.4 we can arrange for $x, y:=\kappa(x)$ to generate a Jacobson-Morosov striple containing $x$. In this $\mathfrak{g}^{y}=\kappa\left(\mathfrak{g}^{x}\right)$, contains $y^{\prime}$ and is non-degenerately paired to $\mathfrak{g}^{x}$ through $K$, so identifies with $\left(\mathfrak{g}^{x}\right)^{*}$.) A basic result proved in [12, Thm. 4.7] is that there exists $h^{\prime} \in \mathfrak{h} \cap \mathfrak{g}^{x}$ making $\left(h^{\prime}, y^{\prime}\right)$ an adapted pair for $\mathfrak{g}^{x}$. Now clearly $K\left(x, \mathfrak{g}^{x}\right)=0$ and so $x \in \mathfrak{k}$. We conclude that for this particular adapted pair the suggestion of 1.4 has a positive answer.

Notice that this construction makes sense for nilpotent orbits generated by a subset of the simple root vectors (called Bala-Carter orbits or orbits of Cartan type) for any simple Lie algebra. However $y^{\prime}$ obtained in this fashion is seldom regular in $\left(\mathfrak{g}^{x}\right)^{*}$ and in fact regularity requires a very careful choice of $\pi^{\prime}$. An interesting case is when card $\pi^{\prime}=1$, 
say $\pi^{\prime}=\{\alpha\}$. Notice that if $\alpha$ is a long root, then $\mathfrak{g}^{x_{\alpha}}$ is conjugate to the centralizer of the highest root vector which is also a standard truncated parabolic subalgebra. Now forgetting type $A_{2 n}$, which is just the case when the Coxeter number is odd, a simple root system contains a distinguished long root defined in terms of its Dynkin diagram. This is the central root in type $A_{2 n+1}$, the root with three neighbours in types $D, E$ and the unique long root with a short root neighbour in types $B, C, F, G$. (For a further interpretation relating this construction to the highest root, see [13, 2.14].)

If one chooses the (long) simple root $\alpha$ as above, then in all cases (except $E_{8}$ ) the element $y^{\prime}$ as defined above can be completed to an adapted pair [12, Sect. 6]; but this generally fails if one takes $\alpha$ to be an arbitrary long simple root. This again verifies our suggestion for that particular pair, showing in addition that the question is rather delicate. In type $E_{8}$, the element $y^{\prime}$ is not regular [12, 6.14] in $\left(\mathfrak{g}^{x}\right)^{*}$ and it is not known if the latter algebra admits an adapted pair. After Yakimova 22] the invariant algebra $Y\left(\mathfrak{g}^{x}\right)$ is not polynomial.

Our suggestion was also found to hold for some adapted pairs for the (truncated) Borel subalgebra in type $A$. In this case the specification of a Borel subalgebra implies a choice of a set $\pi$ of simple roots and it was found that the regular nilpotent element in the conclusion of the suggestion was obtained from $\sum_{\alpha \in \pi} x_{-\alpha}$ through conjugation by a rather carefully chosen element of the Weyl group $W:=N_{G}(H) / H$. It turned out that this element of $W$ made sense for all simple Lie algebras and through its use we were able to construct [13, Thm. 9.4] an algebraic slice for a truncated Borel in all types except $C, B_{2 n}, F_{4}$ even though an adapted pair does not exist (outside type $A$ ). Here the base point $\eta$ was not regular but still satisfied the conclusion of Suggestion 1.4. Obviously we should like to take these last observations further.

We remark that the index of a (truncated) parabolic (resp. biparabolic) was calculated in [5] (resp. [7]) and that in most cases (all cases for types $A, C$ ) the invariant algebra was shown to be polynomial (4], [7]). In type $A$ an adapted pair was constructed for all truncated biparabolics [11. For a centralizer $\mathfrak{g}^{x}$ of a simple Lie algebra $\mathfrak{g}$ the invariant algebra was shown [18 to be polynomial in many cases (all cases in types $A, C$ ), whilst in type $A$, or for a long root vector outside type $E_{8}$, the above construction of an adapted pair (which has the additional property of being "compatible") allows one to prove very easily [12, Thm. 3.5] the polynomiality of $Y\left(\mathfrak{g}^{x}\right)$.

1.6. The purpose of the present article is to verify our suggestion for (truncated) biparabolics of index one. As noted in [9, 2.2,2.3], these are described as the derived algebras of maximal parabolic subalgebras in type $A$ for which the Levi factor consists of two blocks of coprime sizes $p, q$. In this we shall take $p<q$ with the smaller block in the top left hand corner. The parabolic is assumed to have Levi factor having these two blocks and with nilradical $\mathfrak{m}$ being the lower left hand corner block and thus is spanned by root vectors in which the "non-compact" simple root, namely $\alpha_{p}$ in the Bourbaki notation 1, Planche $\mathrm{I}$ ], occurs with coefficient -1 in every root of $\mathfrak{m}$. The truncated parabolic $\mathfrak{p}$ is just the derived algebra of the above. (Though it might be more appropriate to denote it by $\mathfrak{p}^{\prime}$, this would just be cumbersome and in any case we do not need to refer to the parabolic itself.) We denote by $P$ the closed subgroup of $G$ with Lie algebra $\mathfrak{p}$. An adapted pair for 
$\mathfrak{p}$ was constructed in 9]. A rather unusual (but easily proven - see 1.8) aspect of the index one case is that such a pair is unique up to conjugation by an element of $P$. Moreover via [8, Cor. 8.7], every regular orbit meets the resulting slice at exactly one point and even transversally (see [13, Prop. 7.8(ii)] for example). This is the exact analogue of the result obtained in the semisimple case by Kostant [15], [16].

1.7. The proof that our suggestion holds in the above case is obtained from the combinatorial analysis given in the following two sections. This turned out to be surprisingly difficult though ultimately we believe the solution is rather elegant. However unlike the Borel case and the case $p=1$, the element in its conclusion is obtained from a standard nilpotent element not just by conjugation through $W$ but rather by an element of the form $n_{w} b$, with $n_{w}$ a representative of $w \in W$ lying in $N_{G}(H)$ and $b \in B$. Moreover we give a recipe for computing $w$, but at present its meaning is unclear.

1.8. Define $\mathfrak{p}$ as in 1.6. Let us recall the construction of an adapted pair for $\mathfrak{p}$ given in [9]. Let $\mathfrak{h}^{\prime}$ denote the set of diagonal matrices lying in $\mathfrak{p}$. One has $\operatorname{dim} \mathfrak{h}^{\prime}=n-2$.

Let $\pi:=\left\{\alpha_{i}\right\}_{i=1}^{n-1}$ be the set of simple roots for $\mathfrak{s l}(n)$ labelled as in Bourbaki [1, Planche I] with respect to the Borel subalgebra $\mathfrak{b}$ being the set of upper triangular matrices of trace zero.

Identify $\mathfrak{p}^{*}$ with $\mathfrak{p}^{-}:=\kappa(\mathfrak{p})$. Recall that we are assuming $\mathfrak{b} \subset \mathfrak{p}^{-}$. The nilradical $\mathfrak{m}$ of $\mathfrak{p}$ is a complement to $\mathfrak{p}^{-}$in $\mathfrak{g}$ and identifies via the Killing form with the kernel of the restriction map $\mathfrak{g}^{*} \rightarrow \mathfrak{p}^{*}$, that is $\mathfrak{m}=\mathfrak{k}$, in the notation of 1.4. Under the present conventions $\mathfrak{m}$ is spanned by those vectors corresponding to roots in which $\alpha_{p}$ appears with a coefficient of -1 . (This convention should be recalled in $2.6 \mathrm{~b}$ ).)

Recall (cf [9, 2.5]) the notion of the Kostant cascade $\mathscr{B}$ (of positive strongly orthogonal roots) defined for any semisimple Lie algebra. For $\mathfrak{s l}(n)$ this is just $\left\{\alpha_{i}+\alpha_{i+1}+, \ldots,+\alpha_{n-i}\right\}_{i=1}^{[(n-1) / 2]}$. In particular $\mathscr{B} \cap \pi \neq \phi$, if and only if $n$ is even and then this intersection is $\left\{\alpha_{n / 2}\right\}$.

By definition, the Levi factor of $\mathfrak{p}$ is isomorphic to $\mathfrak{s l}(p) \times \mathfrak{s l}(q)$. Let $-\mathscr{B}^{\prime}$ denote its Kostant cascade.

Since $q, p$ are coprime, exactly one of the integers $p, q, n$ is even and so $\left(\mathscr{B} \cup \mathscr{B}^{\prime}\right) \cap(\pi \cup-\pi)$ consists of exactly one element, say $\alpha$. Set $\overline{\mathscr{B}}:=\mathscr{B} \cup \mathscr{B}^{\prime} \backslash\{\alpha\}$. One may remark that up to signs $\mathscr{B} \cup \mathscr{B}^{\prime}$ is a choice of simple roots [9, 2.6]. In particular $\mathscr{B} \cup \mathscr{B}^{\prime}$ is a basis for $\mathfrak{h}^{*}$.

A main result of $[9]$ is that

$$
\eta:=\sum_{\beta \in \overline{\mathscr{B}}} x_{\beta},
$$

is regular (see [9, 3.7]) in $\mathfrak{p}^{*}$ and that there exists a unique $h \in \mathfrak{h}^{\prime}$ such that $h \eta=-\eta$, with respect to co-adjoint action. Thus $(h, \eta)$ is an adapted pair for $\mathfrak{p}$.

It is checked in [9, 3.7] that $\mathbb{K} x_{\alpha}$ is a complement to $\mathfrak{p} \eta$ in $\mathfrak{p}^{*}$. It is further checked [9, 3.3] that $h x_{\alpha}=m x_{\alpha}$, where $m+1$ is the degree, namely $\frac{p^{2}+q^{2}+p q-1}{2}$, of the unique homogeneous generator $f$ of $Y(\mathfrak{p})$. Moreover $f$ is irreducible. Indeed otherwise it could not be the generator of $Y(\mathfrak{p})$. We remark that for $p=1$ there is a rather precise and elegant description of $f$ discovered independently by Dixmier and Joseph (see [4, 15] and 
references therein). However even for the case $p=2, q=3$ a simple description of $f$ is not known.

Let $\mathscr{N}(\mathfrak{p})$, or simply $\mathscr{N}$, denote the zero set of $f$ in $\mathfrak{p}^{*}$. By the above remarks $\mathscr{N}$ is an irreducible closed subvariety of codimension one in $\mathfrak{p}^{*}$. In more general terms if $\mathfrak{a}$ is an algebraic Lie algebra, then $\mathscr{N}(\mathfrak{a})$ is defined to be the nilfibre of the categorical quotient map $\mathfrak{a}^{*} \rightarrow \mathfrak{a}^{*} / / A$. It is seldom irreducible even for a truncated biparabolic [11, 1.4].

The relation $h \eta=-\eta$, forces $P \eta \subset \mathscr{N}$. Since $P \eta$ has codimension index $\mathfrak{p}=1$ in $\mathfrak{p}^{*}$, it follows from the above that $P \eta$ is open dense in $\mathscr{N}$ and consequently $P \eta=\mathscr{N}_{\text {reg }}$. In particular if $\left(h^{\prime}, \eta^{\prime}\right)$ is a second adapted pair for $\mathfrak{p}$, then $\eta^{\prime} \in P \eta$. Moreover since $h$ is uniquely determined by $\eta$, we conclude that there exists $p \in P$ such that $p h=h^{\prime}, p \eta=\eta^{\prime}$.

Through the identifications made in 1.4 we may consider $\eta$ as an element of $\mathfrak{g}^{*}$. Then the relation $h \eta=-\eta$ forces $\eta \in \mathscr{N}(\mathfrak{g})$, but one cannot conclude that $P \eta \subset \mathscr{N}(\mathfrak{g})$. Again $\eta$ is not regular in $\mathscr{N}(\mathfrak{g})$. Here one should stress that by identifying $\mathfrak{p}^{*}$ with $\kappa(\mathfrak{p})$ we are choosing a particular pre-image of $\eta \in \mathfrak{g}^{*}$.

The main result of the present paper is that there exists $\xi \in \mathfrak{m}$ such that $\eta+\xi \in \mathscr{N}(\mathfrak{g})_{\text {reg }}$. This can be expressed as saying that there is some pre-image of $\eta \in \mathfrak{g}^{*}$ lying in $\mathscr{N}(\mathfrak{g})_{\text {reg }}$. We do not believe that this to be a priori obvious. However one may remark that since index $\mathfrak{p}=1$, it follows that $P \eta+\mathfrak{m}$ has codimension 1 in $\mathfrak{g}^{*}$. On the other hand since $\mathfrak{g}$ is semisimple the codimension of $\mathfrak{g}^{*} \backslash \mathfrak{g}_{\text {reg }}^{*}$ in $\mathfrak{g}^{*}$ is 3 (as is well-known - see [10, 2.6.14], for example). It follows that $(P \eta+\mathfrak{m}) \cap \mathfrak{g}_{\text {reg }}^{*} \neq \phi$, however this is not quite what we require.

1.9. Recall the notation of 1.5. Set $x=\sum_{\alpha \in \pi} x_{\alpha}$. Set $\mathfrak{n}^{\prime}=[\mathfrak{n}, \mathfrak{n}]$. We need the following well-known technical result. We give a proof for completeness.

Lemma. $N x=x+\mathfrak{n}^{\prime}$.

Proof. The inclusion $N x \subset x+\mathfrak{n}^{\prime}$, is trivial.

The converse will be proved by an easy induction. For all $\beta \in \Delta^{+}$, we may write $\beta=\sum_{\alpha \in \pi} k_{\alpha} \alpha$ and we set $o(\beta)=\sum_{\alpha \in \pi} k_{\alpha}$. Let $N_{\beta}$ be the closed subgroup of $N$ with Lie algebra $\mathbb{K} x_{\beta}$. For all $k \in \mathbb{N}^{+}$, set

$$
N^{k}:=\prod_{\beta \in \Delta^{+} \mid o(\beta) \geq k} N_{\beta}
$$

Clearly $N^{k}$ is a closed subgroup of $N$ with Lie algebra

$$
\mathfrak{n}^{k}:=\sum_{\beta \in \Delta^{+} \mid o(\beta) \geq k} \mathbb{K} x_{\beta}
$$

Set

$$
\mathfrak{n}_{k}:=\sum_{\beta \in \Delta^{+} \mid o(\beta)=k} \mathbb{K} x_{\beta}
$$

Then

$$
\mathfrak{n}^{k}=\sum_{\ell \geq k} \mathfrak{n}_{\ell}
$$


Suppose we have shown that $x+\mathfrak{n}^{k+1} \subset N^{k} x$, which is of course trivial for $k$ sufficiently large. If $k=1$, we are done. Otherwise use of the well-known relation $\mathfrak{n}_{k}=\left[\mathfrak{n}_{1}, \mathfrak{n}_{k-1}\right]$, together with the induction hypothesis gives the assertion for $k$ replaced by $k-1$.

Remark 1. It is clear that $N x$ is dense in $x+\mathfrak{n}^{\prime}$. On the other hand $N$ is unipotent group acting linearly on its Lie algebra $\mathfrak{n}$. Thus $N x$ is closed in $\mathfrak{n}$ by a result of Rosenlicht [20, Thm. 2] and from this the lemma follows. Actually Rosenlicht attributes (without reference) the required version of the result to Kostant the latter having given a "complicated Lie algebra argument", of which we believe the above is an extract (see [15, Thm. $3.6])$.

Remark 2. The result is even easier for $\mathfrak{s l}(n)$ since closure is not needed. Take $x^{\prime} \in x+\mathfrak{n}^{\prime}$ and let $V$ be the standard $\mathfrak{s l}(n)$ module of dimension $n$. Choose a basis in $V$ so that $x$ has Jordan block form. From this one immediately verifies that $V, x^{\prime} V, x^{\prime 2} V, \ldots$, is a complete flag and so there exists a basis for $V$ such that $x^{\prime}$ has Jordan block form. Thus $x^{\prime}$ (as well as $x$ ) is regular. Consequently $\operatorname{dim} N x^{\prime}=\operatorname{dim} N-\operatorname{dim} C_{N}\left(x^{\prime}\right) \geq \operatorname{dim} N-\operatorname{dim} C_{G}\left(x^{\prime}\right)=$ $|\Delta|-\operatorname{rank} \mathfrak{g}=\operatorname{dim} \mathfrak{n}^{\prime}$. Thus $N x^{\prime}$ must be dense in the irreducible variety $x+\mathfrak{n}^{\prime}$ and hence open. As a special case, $N x$ is open dense in $x+\mathfrak{n}^{\prime}$. Consequently these orbits must meet and so $x^{\prime} \in N x$.

1.10. Let $\mathscr{P}$ denote the set of pairs of coprime positive integers and $\mathscr{S}$ the set of all finite ordered sequences of ones and minus ones. Our construction gives a map (possibly surjective) of $\mathscr{P}$ into $\mathscr{S}$, through the signature of a meander (see 2.1 and 3.3). We believe this to be quite new though whether it has any arithmetical significance is another matter. It would be interesting to determine the image and fibres of this map.

1.11. V. Popov has informed us of work of particularly the Russian school on algebraic and rational slices. Although this has practically no intersection with our present paper (being concerned mainly with case where $\mathfrak{g}$ is a reductive group acting on a finite dimensional module $V$ ) it is nevertheless appropriate to give a sketch of their results of which 19. provides in particular a useful summary.

Adopting the terminology of [19] we call a linear action of a Lie algebra $\mathfrak{a}$ on a finite dimensional vector space co-regular if the algebra of invariant regular functions on $V$ is polynomial.

What we call an algebraic slice in [13, 7.6], the Russian school had called a Weierstrass section. (This terminology comes for the case $\mathfrak{g}=\mathfrak{s l}(3)$ acting on a simple ten dimensional module for which such a section was exhibited by Weierstrass.) The existence of a Weierstrass section (trivially) forces the action to be co-regular.

A fairly comprehensive study of Weierstrass sections was given in [19, Sect 2] for a coregular action of a semisimple Lie algebra $\mathfrak{g}$ acting on a finite dimensional vector space $V$ particularly if either $\mathfrak{g}$ or $V$ is simple. A notable general result [19, Thm. 2.2.15] is that a Weierstrass section exists if the zero fibre $\mathscr{N}_{V}(\mathfrak{g})$ of the categorical quotient map admits 
a regular element (in a sense analogous 1.1). Moreover the converse holds if the set of non-regular elements in $V$ is of codimension $\geq 2$.

The above result and the theorems leading up to it were partly inspired by the seminal work of Kostant [15, 16] as was our own work. Though these are stated for just $\mathfrak{g}$ semisimple it is not improbable that they extend to the general case as we already partly verified in [14. Moreover it is interesting to note that in all our examples (with $\mathfrak{g}$ solvable) where we found [13] a Weierstrass section for which the base point was not regular, the set of non-regular elements (in $\mathfrak{g}^{*}$ ) was indeed of codimension 1 .

Apart from these general considerations, when in comes to actually finding a Weierstrass section the results reported in [19] and own own work [11, 12, 13] are of a quite different nature not least because they are mainly obtained on a case by case basis and whilst [19, Sect 2] concentrates on the semisimple case, our own work concerns the non-reductive case. Indeed it is not easy to find coregular actions, rather difficult to find regular elements in the zero fibre and even harder to exhibit Weierstrass sections if no such elements exist. Just to exemplify the last of these, Popov [19, 2.2.16] notes that for the action $\mathfrak{s l}(n)$ on $n$ copies of its defining $n$ dimensional module, the invariant algebra is generated by the (obvious) determinant and as a consequence the nilfibre has no regular elements, whilst a Weierstrass section obtains by sending all off-diagonal entries to zero and all diagonal entries besides the first to zero. On the other hand our examples [13] come from truncated Borels of simple Lie algebras outside types $A, C, B_{2 n}, F_{4}$ and this for the adjoint action. In these cases they are many generators and a Weierstrass section is not so easy to describe.

Classifying Weierstrass sections for co-regular actions of non-reductive groups is a wide open problem.

Acknowledgement. The authors would like to thank Anna Melnikov for Latex instruction and Vladimir Popov for his comments on some points in the manuscript.

A preliminary version of this result was presented by Florence Fauquant-Millet at the Workshop "Problems and Progress in Lie Algebraic Theory " held on 7-8 July 2010 in the Weizmann Institute.

\section{The Combinatorial Construction}

2.1. Throughout $\mathfrak{g}$ denotes the simple Lie algebra $\mathfrak{s l}(n)$, with $n>2$. Let $\mathfrak{h}$ denote the diagonal matrices in $\mathfrak{g}$. It is a Cartan subalgebra. Let $($,$) denote the Cartan scalar$ product on $\mathfrak{h}$.

Set $I=\{1,2, \ldots, n-1\}, \hat{I}=I \cup\{n\}$. Following Bourbaki [1, Planche I], we choose an orthonormal basis $\varepsilon_{i}: i \in \hat{I}$ in $\mathbb{R}^{n}$ and set $\alpha_{i}=\varepsilon_{i}-\varepsilon_{i+1}: \forall i \in I$. Then $\pi=\left\{\alpha_{i}\right\}_{i \in I}$ is a simple root system for $\mathfrak{g}$.

Let $p, q$ be positive integers with sum $n$. We assume that $p \leq q$. Following a suggestion (see [9, 2.6, Remark] of G. Binyamini we use the Dergachev-Kirillov meanders on the set $\left\{\varepsilon_{1}, \varepsilon_{2}, \ldots, \varepsilon_{n}\right\}$ to describe the support of the second element $\eta$ of the adapted pair $(h, \eta)$ defined in 1.8. This is instead of using the action of the group $\langle i, j\rangle$ defined in [4] (see [9, $2.2]$ ) on the set $\pi:=\left\{\alpha_{1}, \alpha_{2}, \ldots, \alpha_{n-1}\right\}$ of simple roots. Here a meander is interpreted as an 
orbit of the group $\Gamma$ generated by involutions $\sigma, \tau$ defined as follows. For all $i=1,2, \ldots, n$, set $\sigma(i)=n+1-i$ and

$$
\tau(i)= \begin{cases}p+1-i & : 1 \leq i \leq p, \\ n+p+1-i & : p+1 \leq i \leq n .\end{cases}
$$

One checks that $\tau \sigma(k)=p+k$, where it is understood that any integer is reduced modulo $n$ so that it lies in $[1, n]$. It follows that $\{1,2, \ldots, n\}$ is a single $\Gamma$ orbit $\mathscr{O}$ if and only if $p, q$ are coprime.

\subsection{Assume from now on that $p, q$ are coprime.}

By a slight abuse of language we say that an end point of $\mathscr{O}$ is an element of $\mathscr{O}$ fixed by either $\sigma$ or $\tau$. One easily checks that $\mathscr{O}$ has exactly two end points $a, b$.

If $p$ is odd, we can set $a=(p+1) / 2$. If in addition $n$ (resp. $q$ ) is odd we can set $b=(n+1) / 2($ resp. $b=p+(q+1) / 2)$. In this case $a<b$. If $p$ is even we can set $b=(n+1) / 2$ and $a=p+(q+1) / 2$. In this case $b<a$. We call $a$ (resp. $b)$ the starting (resp. finishing) point of $\mathscr{O}$.

We define a bijection $\varphi: \hat{I} \stackrel{\sim}{\rightarrow} \hat{I}$ as follows. First note that the starting point $a$ is always a $\tau$ fixed point. Then set $\varphi(1)=a, \varphi(2)=\sigma(a), \varphi(3)=\tau \sigma(a), \ldots$ (This may be a little confusing since the domain which identifies with $\{1,2, \ldots, n\}$ and the target which identifies with $\mathscr{O}$ are both denoted by $\hat{I}$.)

Set $\beta_{i}=\varepsilon_{\varphi(i)}-\varepsilon_{\varphi(i+1)}: i \in I$. By our conventions $\beta_{1}$ is a positive (resp. negative) root if $p$ odd (resp. even). Again $\left(\beta_{i}, \beta_{i+1}\right)<0$, for all $i \in I \backslash\{n-1\}$, whilst the remaining scalar products between distinct elements, vanish. Hence $\Pi:=\left\{\beta_{i}\right\}_{i \in I}$ is a simple root system and in particular $W$ conjugate to $\pi$.

Recall 1.8. One easily checks that up to signs there is a unique subset of $\Pi$ which is the Kostant cascade $\mathscr{B}$ for $\mathfrak{s l}(n)$ and again up to signs $\Pi \backslash \mathscr{B}$ is the opposed Kostant cascade $\mathscr{B}^{\prime}$ for the Levi factor $\mathfrak{s l}(p) \times \mathfrak{s l}(q)$ of $\mathfrak{p}$. In particular relative to $\pi$ the elements of $\mathscr{B}$ (resp. $\left.\mathscr{B}^{\prime}\right)$ are positive (resp. negative roots). It is the analysis of these signs which is the main combinatorial content behind the construction of a further simple root system $\Pi^{*}$. This is the main step in achieving our goal of finding a regular nilpotent element $y$ of $\mathfrak{g}$, whose restriction to $\mathfrak{p}$ is $\eta$.

2.3. Towards the above goal we define a turning point of $\mathscr{O}$ to be an element $\varphi(t): t \in \hat{I}$ such that $t-\sigma(t)$ is of opposite sign to $t-\tau(t)$. Here we include the end points of $\mathscr{O}$ in its set of turning points, that is to say when one of the above integers is zero. The remaining turning points are called internal turning points.

The observation in 2.2 can be expressed as saying that for all $i \in I$ one has either $\beta_{i} \in \mathscr{B} \cup \mathscr{B}^{\prime}$ or $\beta_{i} \in-\left(\mathscr{B} \cup \mathscr{B}^{\prime}\right)$. Notice further that up to signs if $\beta_{i-1} \in \mathscr{B}$, then its successor $\beta_{i} \in \mathscr{B}^{\prime}$ and vice-versa. Let us now make precise how these signs vary. Indeed taking account of the fact that the elements of $\mathscr{B}$ (resp. $\mathscr{B}^{\prime}$ ) are positive (resp. negative) roots, the following fact is easily verified.

Lemma. Suppose $\beta_{t-1} \in \mathscr{B} \cup \mathscr{B}^{\prime}\left(\right.$ resp. $\left.\beta_{t-1} \in-\left(\mathscr{B} \cup \mathscr{B}^{\prime}\right)\right)$, then $\beta_{t} \in-\left(\mathscr{B} \cup \mathscr{B}^{\prime}\right)$ (resp. $\left.\beta_{t} \in \mathscr{B} \cup \mathscr{B}^{\prime}\right)$ if and only if $\varphi(t)$ is an internal turning point of $\mathscr{O}$. 
Remark. By our conventions if $p$ is odd, then $\beta_{1} \in \mathscr{B}$ and if $p$ is even, then $\beta_{1} \in-\mathscr{B}$.

2.4. It is easy to compute the set of turning points of $\mathscr{O}$. They form two disjoint "connected" sets, namely $A:=[[p / 2]+1, p]$ and $B:=[[n / 2]+1, p+[(q+1) / 2]]$. If $p$ is odd both have cardinality $(p+1) / 2$ and moreover $a \in A$ and $b \in B$. If $p$ is even, then $A$ has cardinality $p / 2$, whilst $B$ has cardinality $1+p / 2$ and contains both $a$ and $b$.

The $\Gamma$ orbit $\mathscr{O}$ viewed as starting at $a$ and finishing at $b$ acquires a linear order with smallest element $a$ and largest element $b$ being the natural order on $\hat{I}$ translated under $\varphi$. It induces a linear order on the set $T$ of turning points.

Lemma. With respect to the above linear order on $T$ the nearest neighbour(s) of an element of $A$ lie(s) in $B$ and vice-versa.

Proof. Since $|A| \leq|B|$ with equality unless both $a, b$ lie in $B$ in which case $|A|+1=|B|$, it is enough to show that the set of successors of an element of $B$ first meets $A$. Take $b^{\prime} \in B$ and assume that $\sigma\left(b^{\prime}\right)$ (resp. $\tau\left(b^{\prime}\right)$ ) is a successor of $b^{\prime}$. Since $B$ lies in an interval of width $\leq p / 2$, whilst $\tau \sigma$ (resp. $\sigma \tau$ ) is translation by $p$ (resp. $-p$ ), it follows that the set of successors of $b^{\prime}$ with respect to powers of $\tau \sigma$ (resp. $\sigma \tau$ ) meets the interval $[1, p]$ before it meets $B$ again.

Finally observe that the image under $\tau$ of any of the above $p$-translates of $b^{\prime}$ (that is to say after starting at $b^{\prime}$ and until $[1, p]$ is reached) do not lie in $B$. On the other hand when $[1, p]$ is reached then the set of successors first meets $A$ since $A \cup \tau(A)=[1, p]$. Hence the assertion of the lemma.

2.5. We can now describe the signs mentioned in 2.3.

In view of 2.4, that there are always $p+1$ turning points of which $p-1$ are internal. If $p$ is odd (resp. even) we label them as $\varphi\left(t_{i}\right): i=1,2, \ldots, p+1$ (resp. $i=0,1, \ldots, p$ ), where the $t_{i}$ are strictly increasing. Set $J:=1,2, \ldots,[(p+1) / 2]$. With this choice and our previous conventions $A=\left\{\varphi\left(t_{2 j-1}\right): j \in J\right\}$.

For all $k=0,1, \ldots, p$, set $\epsilon_{i}=(-1)^{k-1}$, for all $i=t_{k}, t_{k}+1, \ldots, t_{k+1}-1$. That is $\epsilon_{i}=1$ (resp. $\epsilon_{i}=-1$ ) in the interval in which an element of $A$ (resp. $B$ ) is followed by an element of $B$ (resp. $A)$.

Corollary. $\mathscr{B} \cup \mathscr{B}^{\prime}=\left\{\epsilon_{i} \beta_{i}: i \in I\right\}$. In particular the $\epsilon_{i} \beta_{i}: i \in I$ which lie in $\mathscr{B}$ (resp. $\left.\mathscr{B}^{\prime}\right)$, are positive (resp. negative) roots.

2.6. Observe that there is exactly one index $i \in I$ such that $\beta_{i} \in \pm \pi$. We call this the exceptional index $e$ and $\beta_{e}$ the exceptional value.

From the corollary we see that $\mathscr{B} \cup \mathscr{B}^{\prime}$ cannot be a simple root system because successive scalar products acquire the wrong sign as the (internal) turning points are crossed. Our aim is to change the $\epsilon_{i} \beta_{i}$ to new elements $\beta_{i}^{*}$, so that

a) $\Pi^{*}:=\left\{\beta_{i}^{*}\right\}_{i \in I}$, is a simple root system,

b) Suppose $\beta_{i}^{*} \neq \epsilon_{i} \beta_{i}$. Then expressed as a sum of elements of $\pi$, the "non-compact" root $\alpha_{p}$ appears in $\beta_{i}^{*}$ with a negative coefficient (hence with coefficient -1 ).

c) $\beta_{e}^{*} \neq \epsilon_{e} \beta_{e}$. 
d) $\epsilon_{i} \beta_{i} \in \mathbb{N}^{*}$, for all $i \in I \backslash\{e\}$. Equivalently the $\epsilon_{i} \beta_{i}: i \in I \backslash\{e\}$ are positive roots with respect to $\Pi^{*}$.

The meaning of these conditions is as follows. Set

$$
y^{\prime}=\sum_{i \in I} x_{\beta_{i}^{*}} .
$$

Condition a) means that $y^{\prime}$ is a regular nilpotent element of $\mathfrak{s l}(n)$ and hence can be conjugated by an element $w$ of the Weyl group $W=S_{n}$ to a standard nilpotent element $y_{0}:=\sum_{\alpha \in \pi} x_{\alpha}$. Condition d) means that the $x_{\epsilon_{i} \beta_{i}}$, for $i$ non-exceptional, either already occur in $y^{\prime}$ or can be added to $y^{\prime}$ as commutators of the $x_{\beta_{i}^{*}}: i \in I$. In particular by Lemma 1.9, the new element $y^{\prime \prime}$ obtained by adding these commutators, namely the $\left\{x_{\epsilon_{i} \beta_{i}}: \epsilon_{i} \beta_{i} \neq \beta_{i}^{*}\right\}_{i \in I \backslash\{e\}}$, is again regular nilpotent. Finally b) and c) imply that $y^{\prime \prime}$ restricted to $\mathfrak{p}$ coincides with $\eta$ as defined in 1.8. Let $B$ denote the Borel subgroup of $G$ defined with respect to $\pi$.

Suppose conditions a)-d) are satisfied. We conclude by the above, Lemma 1.9 and Corollary 2.5 that there exists $w \in W$ and $b \in B$ such that the restriction of $y:=n_{w} b y_{0}$ is $\eta$. Moreover we shall give a (fairly) explicit expression for $\Pi^{*}$ and this determines $w$.

Since $P$ contains the opposed Borel subgroup $B^{-}$rather than $B$ one should consider $y_{0}$ defined above as the negative element of a principal s-triple. In the present work this has no particular significance.

The construction of $\Pi^{*}$ and the proof that it satisfies a)-d) above is given in the next section. The proof is illustrated by Figures 1-7. It should also be possible for the reader to reconstruct the analysis from just these figures.

Remarks. One could imagine that a simpler way to satisfy these conditions might be possible by the following approach. Recall that the $\epsilon_{i} \beta_{i}: i \in I$ form a basis for $\mathfrak{h}^{*}$. Thus we can choose $c_{i} \in \mathbb{N}^{+}: i \in I$ such that there is a unique element $h \in \mathfrak{h}$ satisfying $h\left(\epsilon_{i} \beta_{i}\right)=c_{i}, \forall i \in I$, and that this element is regular. Then $\Delta^{*+}:=\{\alpha \in \Delta \mid h(\alpha)>0\}$ is a choice of positive roots for $\Delta$ and so defines a set $\Pi^{*}$ of simple roots in which $\mathrm{d}$ ) will be satisfied by construction and even in the overly strong form that $\epsilon_{i} \beta_{i} \in \mathbb{N}^{*}$, for all $i \in I$. It is not so obvious if and how we can choose the $c_{i}: i \in I$, to ensure that $\mathrm{b}$ ) is satisfied. Again c) will not be satisfied in general; but in our approach we modify our solution weakening this overly strong form of d) to recover condition c). A postiori one may recover a good choice of the $c_{i}: i \in I$ by setting $h(\alpha)=1, \forall \alpha \in \Pi^{*}$.

2.7. Relative to $\pi$, the roots in $\mathscr{B}^{\prime}$ have a zero coefficient of $\alpha_{p}$. Thus by Corollary 2.5, all elements of $\left\{\epsilon_{i} \beta_{i}\right\}_{i \in I}$ have a non-negative coefficient of $\alpha_{p}$, which is hence in $\{0,1\}$. This coefficient is non-zero only if $\beta_{i} \in \pm \mathscr{B}$. By our conventions (see 2.2) $\beta_{i} \in \pm \mathscr{B}$, if and only if $i$ is odd. Thus $\beta_{i}$ has a non-zero coefficient of $\alpha_{p}$ only if $i$ is odd. In particular neighbours $\beta_{i}, \beta_{i+1}$ cannot both have a non-zero coefficient of $\alpha_{p}$.

Fix $t \in I$. Call $t$ a nil point if the coefficient of $\alpha_{p}$ in $\beta_{t}$ is non-zero and a boundary point if $\varphi(t)$ or $\varphi(t+1)$ is a turning point. Call $\beta_{t}$ a boundary (resp. nil) value if $t$ is a boundary (resp. nil) point. (By Corollary 2.5 and our convention in 1.6 it follows that $\beta_{t}$ is a nil value if and only if $x_{\epsilon_{t} \beta_{t}}$ belongs to the nilradical of $\mathfrak{p}^{-}$.) 
The unique boundary value to an end point is called an end value.

\section{Lemma.}

(i) Suppose $\varphi(t)$ is an internal turning point, then $t$ and $t-1$ cannot be both nil points.

(ii) Suppose $t \in I$ is a nil boundary point with $\varphi(t) \in B($ resp. $\varphi(t+1) \in B)$. Then $\varphi(t+1) \in A(\operatorname{resp} . \varphi(t) \in A)$.

(iii) Suppose $\varphi(t) \in A$. Then $t-1$ or $t$ must be a nil point, in particular 1 must be a nil point if $p$ is odd.

Proof. (i) follows from the remarks in the first paragraph above.

(ii) Since $t$ is a nil point, we must have $\sigma(\varphi(t))=\varphi(t+1)$. Now suppose $i:=\varphi(t) \in B$. Then $i \geq[n / 2]+1$. Thus $t$ is a nil point if and only if $\sigma(i) \leq p$. Again $i \leq p+[(q+1) / 2]$ so $\sigma(i)=n+1-i \geq[q / 2]+1 \geq[p / 2]+1$. Consequently $\varphi(t+1)=\sigma(i) \in A$, as required. The proof of the second case is exactly the same.

(iii) Since $i:=\varphi(t) \in A$, we have $i \leq p$ and so $\sigma(i) \geq p+1$. Thus either $\beta_{t}$ or $\beta_{t-1}$ must have a non-zero coefficient of $\alpha_{p}$. Hence (iii).

Remarks. Since $\beta_{i}=\varepsilon_{\varphi(i)}-\varepsilon_{\varphi(i+1)}$ we may regard $\beta_{i}$ as lying between the elements $\varphi(i), \varphi(i+1)$ of $\varphi(\hat{I})$. We say that $\beta_{i-1}, \beta_{i}$ are the neighbours of $\varphi(i): i \in \hat{I}$ in $\pm\left(\mathscr{B} \cup \mathscr{B}^{\prime}\right)$ and that $\varphi(i), \varphi(i+1): i \in I$ are the neighbours of $\beta_{i} \in \pm\left(\mathscr{B} \cup \mathscr{B}^{\prime}\right)$. Then (iii) of the lemma can be expressed as saying that every element of $A$ has exactly one nil boundary value neighbour, whereas (ii) of the lemma can be expressed as saying that if a nil boundary value has an element of $B$ as a neighbour, then it is sandwiched between an element of $A$ and an element of $B$. By (ii) and (iii) of the lemma every nil boundary value has a unique element of $A$ as a neighbour. Finally an end value is non-nil only if it is the (unique) neighbour of an element of $B$. For example if $p=2, q=5$, both end-points are non-nil. However an end value can be nil even if it is a neighbour of an element of $B$. For example if $p=2, q=3$, the starting value is nil.

2.8. Intervals. Let $\varphi(s), \varphi(t) \in T$ be turning points with $s<t$. The subset $I_{s, t}:=$ $\{s, s+1, \ldots, t-1\}$ is called an interval. If $\varphi(t)$ is the immediate successor to $\varphi(s)$ in $T$, it is called a simple interval. Otherwise it is called a compound interval.

The sum

$$
\iota_{s, t}:=\sum_{i \in I_{s, t}} \beta_{i}
$$

is called a simple (resp. compound) interval value if $I_{s, t}$ is simple (resp. compound). The set $\left\{\beta_{i}: i \in I_{s, t}\right\}$ is called the support of $\iota_{s, t}$ or of $I_{s, t}$.

Lemma. Let $I_{r, s}$ be a simple interval. There is exactly one $i \in I_{r, s}$ such that $\beta_{i}$ has a non-zero coefficient of $\alpha_{p}$.

Proof. Observe that

$$
\iota_{r, s}=\varepsilon_{\varphi(r)}-\varepsilon_{\varphi(s)} .
$$

Since $\varphi(r) \in A$ and $\varphi(s) \in B$ or vice-versa, it follows from 2.4 that the coefficient of $\alpha_{p}$ in the above sum equals one or minus one. Moreover there can be no cancellations of 
coefficients of $\alpha_{p}$ in the sum because only alternate terms can have a non-zero coefficient and these are either all positive roots or all negative roots since the indices lie between successive turning points. Hence the assertion.

2.9. The Sign of Simple Interval Values. Take $\varphi(s) \in T$ and let $\varphi(t)$ be its immediate successive in $T$. The sign of the simple interval value $\iota_{s, t}$ is said to be positive (resp. negative) if $\varphi(s) \in A$ (resp. $\varphi(s) \in B$ ).

A positive (resp. negative) interval value is a positive (resp. negative) root relative to $\pi$ with the coefficient of $\alpha_{p}$ being 1 (resp. -1 ), by the definition of the $\epsilon_{i}$, Corollary 2.5] and Lemma 2.8.

2.10. The Exceptional Value. Recall the exceptional value $\beta_{e}: e \in I$ defined in 2.6. It defines a unique simple root $\alpha \in \pi$ and one has $\beta_{e}=\alpha$, up to a sign.

Lemma. The exceptional value $\beta_{e}$ is never a nil value, equivalently $\alpha \neq \alpha_{p}$. It is a boundary value to some unique turning point, which is either

(i) internal,

or

(ii) an end point lying in $B$.

Proof. Since $p, q$ are coprime, exactly one of the integers $p, 2 p+q, n=p+q$, call it $m$, is even. Then $\beta_{e}= \pm \alpha_{m / 2}$. For this to be a nil value we would need $\alpha_{m / 2}=\alpha_{p}$, that is $m / 2=p$, which is impossible since $p<n / 2$. This proves the first assertion.

One checks from the description of the turning points in 2.4 that either $m / 2$ or $1+m / 2$ is a turning point. They cannot both be turning points because then $\beta_{e}$ would be nil by 2.8, contradicting the first part. On the other hand by Lemma 2.7(iii) an end-point lying in $A$ is nil. Hence the second assertion.

Remark. On may check from 2.2, that (ii) holds if and only if $p=1$.

2.11. Isolated values. We call $t \in I$ an isolated point if both $\varphi(t)$ and $\varphi(t+1)$ are turning points. By Lemma 2.8 an isolated point is necessarily nil. If $t$ is an isolated point we call $\beta_{t}$ an isolated value.

\section{The Description of $\Pi^{*}$}

3.1. Recall that we aim to construct $\Pi^{*}$ by changing some of the $\epsilon_{i} \beta_{i}$. Here it is convenient to write $\beta_{i}^{*}=\epsilon_{i} \beta_{i}^{\prime}$ and to say that a value is changed if $\beta_{i}^{\prime} \neq \beta_{i}$. Let us describe those values that are changed. Here we impose three general rules. The first two are

1) Change only boundary values and change only those which are non-nil.

2) Change exactly one of the boundary values at each internal turning point.

Remark. In the initial stage (up to 3.8) end values will not be changed. However if an end value is exceptional (and hence non-nil and so the unique neighbour of an element of $B)$, then it will be changed in the final stage (3.9). 
3.2. To describe our third general rule we need the following preliminary. For the moment we ignore the exceptional value.

For all $i, j \in \hat{I}$, with $i<j$, set $\beta_{i, j}=\varepsilon_{\varphi(i)}-\varepsilon_{\varphi(j)}$, which we recall is a root (and positive with respect to $\Pi$ ). In this notation $\beta_{i}=\beta_{i, i+1}$. Again if $\varphi(i), \varphi(j) \in T$, we have $\beta_{i, j}=\iota_{i, j}$.

Now let $\beta_{i}$ be a boundary value which is to be changed (according to rules 1) and 2) - in particular $\beta_{i}$ is a non-nil boundary value). Then there is an internal turning point, say $\varphi\left(t_{s}\right)$ which either equals $\varphi(i+1)$ or $\varphi(i)$. (Both possibilities cannot simultaneously arise since otherwise by Lemma 2.8, $\beta_{i}$ would be a nil boundary value.) In the first (resp. second) case we shall say that $\beta_{i}$ is above (resp. below) $\varphi\left(t_{s}\right)$.

In the first case we replace $\beta_{i}$ by $\beta_{i}^{\prime}$ defined by adding to $\beta_{i}$ "a compound interval value which is an odd sum of simple interval values below $\varphi\left(t_{s}\right)$ ", that is to say we set

$$
\beta_{i}^{\prime}=\beta_{i}+\beta_{t_{s}, t_{r}}=\beta_{t_{s}-1, t_{r}}: r-s \in 2 \mathbb{N}+1 .
$$

In the second case we replace $\beta_{i}$ by $\beta_{i}^{\prime}$ defined by adding to $\beta_{i}$ "a compound interval value which is an odd sum of simple interval values above $\varphi\left(t_{s}\right)$ ", that is to say we set

$$
\beta_{i}^{\prime}=\beta_{i}+\beta_{t_{r}, t_{s}}=\beta_{t_{r}, t_{s}+1}: s-r \in 2 \mathbb{N}+1 .
$$

We may summarize the above by saying that in both cases the added interval value is on the opposite side of the turning point to the element in question and is a sum of an odd number of simple interval values.

Finally (recall) that we set $\beta_{i}^{*}=\epsilon_{i} \beta_{i}^{\prime}$.

Lemma. Suppose $\beta_{i}^{\prime} \neq \beta_{i}$. Then $\epsilon_{i} \beta_{i}^{\prime}$ is a root. Moreover expressed as a sum of elements of $\pi$, the non-compact root $\alpha_{p}$ has coefficient -1 in $\epsilon_{i} \beta_{i}^{\prime}$.

Proof. Since $\beta_{i}$ is a non-nil boundary value the coefficient of $\alpha_{p}$ in it is zero.

By definition $\epsilon_{i}$ changes sign as each turning point is crossed. Thus if $\epsilon_{i}=1$ (resp. $\epsilon_{i}=-1$ ). then the nearest simple interval value added to $\beta_{i}$ is negative (resp. positive). Then by the second paragraph of 2.9 the coefficients of $\alpha_{p}$ of the successive simple interval values added to $\epsilon_{i} \beta_{i}$ are $\{-1,1,-1, \ldots\}$, whereas by construction the number of such simple intervals is odd.

3.3. Signature. To complete our description of $\Pi^{*}$ we must now specify which boundary values are to be changed (which will specify $t_{s}$ and how $t_{r}$ in equations $(*)$ and $(* *)$ is determined).

The above data will be completely determined by the signature of the orbit $\mathscr{O}$ defined as follows.

Recall that by the choices made in 2.5 and by Lemma 2.7, at each turning point $\varphi\left(t_{2 j-1}\right)$ : $j \in J$, which we recall lies in $A$, one has that either $\beta_{t_{2 j-1}-1}$ or $\beta_{t_{2 j-1}}$ is nil (but not both). In the first case we set $\operatorname{sg}(j)=-1$ (to specify that the nil boundary value is above the turning point) and in the second case we set $\operatorname{sg}(j)=1$ (to specify that the nil boundary value is below the turning point).

If $\operatorname{sg}(1)=1$ (which is always the case if $p$ is odd) then there is a unique increasing sequence $j_{1}, j_{2}, \ldots, j_{r} \in J$ with $j_{1}=1$, such that

$$
\operatorname{sg}(i)=(-1)^{u-1}, \forall i=j_{u}, j_{u}+1, \ldots, j_{u+1}-1, \forall u=1,2, \ldots, r-1, \quad \operatorname{sg}\left(j_{r}\right)=(-1)^{r-1} .
$$


If $\operatorname{sg}(1)=-1$ (which can be the case if $p$ is even) then there is a unique increasing sequence $j_{1}, j_{2}, \ldots, j_{r} \in J$ with $j_{1}=1$, such that

$$
\operatorname{sg}(i)=(-1)^{u}, \forall i=j_{u}, j_{u}+1, \ldots, j_{u+1}-1, \forall u=1,2, \ldots, r-1, \quad \operatorname{sg}\left(j_{r}\right)=(-1)^{r} .
$$

We say that the signature at the turning point $\varphi\left(t_{2 j-1}\right) \in A$ is positive (resp. negative) if $\operatorname{sg}(j)=1($ resp. $\operatorname{sg}(j)=-1)$.

Finally the signature of $\mathscr{O}$ is defined to be the set $\{s g(i)\}_{i=1}^{[p / 2]}$. In the notation of 1.10 , it lies in $\mathscr{S}$ and defines a map of the set of coprime pairs $\mathscr{P}$ into $\mathscr{S}$.

3.4. We assume until the end of [3.9, that the signature at the first turning point in $A$ is positive. This is always the case if $p$ is odd by virtue of Lemma 2.7(iii). Then the easiest case to describe is when there are no signature changes. This is illustrated in Figure 1, where the given pattern is repeated as many times as there are turning points in $A$.

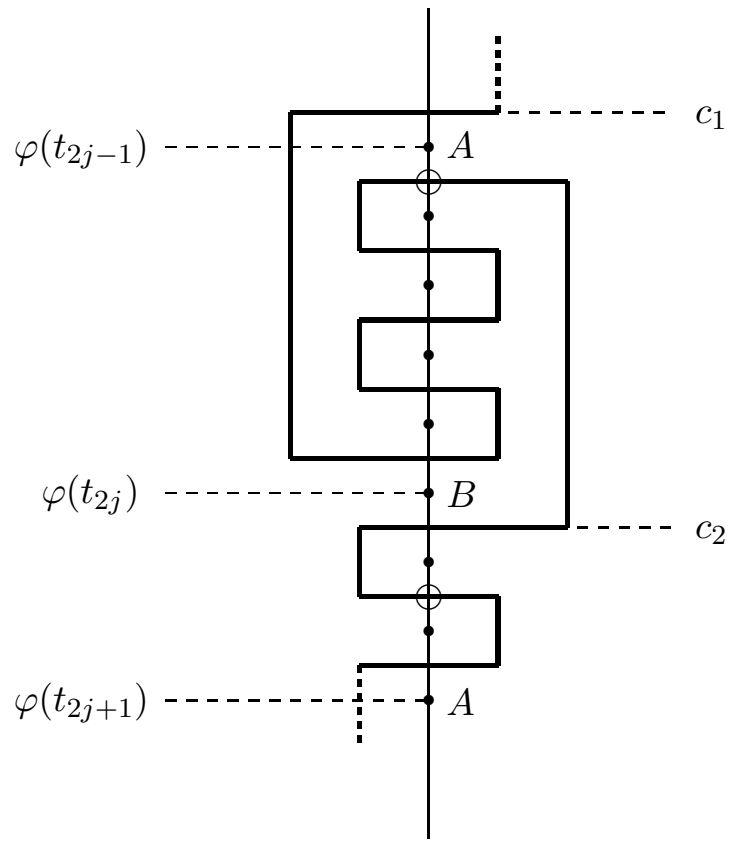

Figure 1.

This shows the basic repeating pattern for positive signature. Turning points are labelled by their type, that is $A$ or $B$, and nil values are encircled. The dots on the vertical central line label a subset of $\hat{I}=\{1,2, \ldots, n\}$. In the language of 3.4, the turning point $\varphi\left(t_{2 j-1}\right) \in A$ has positive signature. In the terminology of 3.6. the thickened lines describe the links between the elements of $\beta_{i}^{*}: i \in I$ and define a sub-chain linking $\beta_{t_{2 j-1}-1}^{*}$ to $\beta_{t_{2 j}}^{*}$. The non-nil boundary values that are changed carry the symbol $c$ which is given the subscript 1 or 2 depending on whether rule 1) or 2) of 3.1 is applied as described in 3.5. The map $\chi$ defined in 3.5 takes $\varphi\left(t_{2 j-1}\right)$ to $\varphi\left(t_{2 j}\right)$. A mirror reflection perpendicular to the vertical axis gives the basic repeating pattern for negative signature. 
The next easiest case is when there is one signature change, namely from positive to negative. After that there is the case when there are two signature changes, namely from positive to negative to positive. From then on it is simply a repetition of the procedure for two signature changes. The first two cases can be considered as a degeneration of the third by simply eliminating terms. Thus it will suffice to describe the case of two signature changes, to make precise what is meant by degeneration and to describe the modification needed if the signature of $\mathscr{O}$ is initially negative which can happen if $p$ is even.

Fix a positive odd integer $u \leq r$ and set $j=j_{u}, k=j_{u+1}, \ell=j_{u+2}$. The first and second cases above correspond to $k$ not being defined and $k$ being defined but $\ell$ not being defined.

Assume $k$ is defined. Then by definition $\varphi\left(t_{2(k-1)-1}\right) \in A$ and admits a nil boundary value just below, namely $\beta_{t}: t=t_{2(k-1)-1}$. If $\ell$ is not defined let $\varphi(s)$ be the last turning point or (end point) $\varphi(n)$, otherwise set $s=t_{2 \ell-2}$. In both cases $\varphi(s) \in B$.

If $t>1$, set

$$
\beta_{t-1}^{\prime}=\beta_{t-1}+\beta_{t, s}=\beta_{t-1, s} .
$$

Observe that $\beta_{t, s}$ is defined even when $t=1$ and is a compound interval value $\iota_{t, s}$ which is a sum of $2 m+1: m=\ell-k$ adjacent simple interval values $\iota_{1}, \iota_{2}, \ldots, \iota_{2 m+1}$, starting at $\iota_{1}=\iota_{t_{2 k-3}, t_{2 k-2}}$.

Suppose $\ell$ is defined (and hence so is $\beta_{s}$ ). If $\iota_{1}$ is not reduced to an isolated value, set

Otherwise set

$$
\beta_{s}^{\prime}=\beta_{s}+\beta_{t, s}=\beta_{t, s+1}
$$

$$
\beta_{s}^{\prime}=\beta_{s}+\sum_{i=3}^{2 m+1} \iota_{i}=\beta_{t_{2 k-1}, s+1} .
$$

3.5. In the remaining cases added interval values will be simple and we only have to specify the non-nil boundary values which are changed. We need only describe these between the turning points $t_{2 j-1}$ and $t_{2 \ell-1}$ in the notation of 3.4 since the pattern just repeats itself. For this there is a simple algorithm.

Notice first that if a simple interval value $\iota$ is to be added to a non-nil boundary value $\beta_{i}$, then this simple interval value is uniquely determined by $\beta_{i}$ itself via the rule in 3.2 .

We define a map $\chi$ from the set of internal turning points of $A$ to the set of turning points of $B$.

At every internal turning point $\varphi(t) \in A$, (so then $t=t_{2 v-1}$ for some $v \in J$ ) there is exactly one non-nil boundary value $\beta_{u}$ and by rule 1) of 3.1, it must be changed, that is $\beta_{u}^{\prime} \neq \beta_{u}$. By the rule described in 3.2 there is a unique turning point $\varphi\left(t^{\prime}\right) \in B$ so that $\beta_{u}^{\prime}=\beta_{u}+\iota_{t, t^{\prime}}$, where we have defined $\iota_{t, t^{\prime}}:=\iota_{t^{\prime}, t}$ if $t^{\prime}<t$. We set $t^{\prime}=\chi(t)$ and $\chi(\varphi(t))=\varphi(\chi(t))=\varphi(t)$. Notice that $t^{\prime}>t$ (resp. $\left.t<t^{\prime}\right)$ if the signature of $\varphi(t) \in A$ is positive (resp. negative) and we say that $\varphi\left(t^{\prime}\right) \in B$ is a subsequent (resp. previous) turning point to $\varphi(t) \in A$.

Observe further that there is a unique boundary value $\beta_{w}$ to $\varphi\left(t^{\prime}\right) \in B$ such that the scalar product $\left(\beta_{u}^{\prime}, \beta_{w}\right)$ is strictly positive. With one possible exception (within a double 
Figure 2.

In the notation of 3.4 this compares the cases when $\iota_{1}$ is not isolated (on the left) and $\iota_{1}$ is isolated (on the right). The same conventions as in Figure 1 apply, where in addition the additional subscript 0 to $c_{1}, c_{2}$ refers to the application of the rule described in 3.4. The particular case here corresponds to taking $\ell=k+1$. For the general case one must extend the two outermost lines in each diagram downwards and insert a further $\ell-(k+1)$ copies of the basic repeating pattern for negative signature. This is illustrated by Figure 3 in which the case $\ell=k+2$ is considered.

We remark that one may have an isolated point in a region of negative signature. This is illustrated in Figure 4.

One checks from 3.4, 3.5 that the map $\chi$ defined above is an injection from the set of internal turning points lying in $A$ to the set of turning points in $B$. In all cases the latter set has cardinality one greater than the former. Thus the cokernel of $\chi$ is a singleton which we call the undecided element $d \in B$. It is clear that the above algorithm just leaves at most one boundary value of $d$ undecided. The exact location of $d$ depends on the signature of $\mathscr{O}$ as we now explain.

Suppose $p$ is odd and recall $\varphi\left(t_{1}\right)$ has positive signature. If $\varphi\left(t_{3}\right)$ is not defined, then $d=\varphi\left(t_{2}\right)$. It is a finishing point in $B$ and we leave its unique neighbour unchanged. If $\varphi\left(t_{3}\right)$ is defined and has positive signature, then $d=\varphi\left(t_{2}\right)$ and we change $\beta_{t_{2}}$ by adding a simple interval value, namely $\iota_{t_{1}, t_{2}}$.

Finally suppose that $\varphi\left(t_{3}\right)$ has a negative signature. This corresponds to having $k=2$ in 3.4. Then in the notation of 3.4 one has $d=\varphi(s)$. If $d$ is a finishing point we leave its unique neighbour unchanged. Otherwise we change $\beta_{s}$ by the rules described in $3.4(* * *)$ or 3.4 $(* *)$, depending on whether $\iota_{1}$ is reduced to an isolated value or not.

One may remark that when $\varphi\left(t_{3}\right)$ is defined the solutions given in the above two paragraphs would result if we were to treat $\varphi\left(t_{1}\right)$ as if it were an internal turning point.

Suppose $p$ is even. If $\varphi\left(t_{3}\right)$ has positive signature, Then $d=\varphi(1)$ and we leave its unique neighbour unchanged. The case when $\varphi\left(t_{3}\right)$ has negative signature will be postponed to 3.10 .

Note that if $\varphi\left(t^{\prime}\right)$ is an end-point, namely $t^{\prime}=1$ (resp. $t^{\prime}=n$ ), its unique boundary value, that is $\beta_{1}$ (resp. $\beta_{n-1}$ ) is left unchanged by the above procedure. However this will be modified in 3.9 .

This (nearly !) completes our description of $\Pi^{*}$. What can happen however is that condition c) of 2.6 can sometimes fail and this will need a further modification to be described in 3.9 .

3.6. Define $\beta_{i}^{\prime}: i \in I$ through the rules described in 3.1 - 3.5. set $\beta_{i}^{*}=\epsilon_{i} \beta_{i}^{\prime}$ and $\Pi^{*}=$ $\left\{\beta_{i}^{*}\right\}_{i \in I}$.

Here we show that $\Pi^{*}$ satisfies condition a) of 2.6 by exhibiting an ordering so that nearest neighbours have a strictly negative scalar product (which we call a link) and that no other non-zero scalar products exists between distinct elements. We call such a succession of links, a sub-chain. 
In this we shall assume that $k$ and $\ell$ of 3.4 are defined, otherwise one just obtains a degeneration of that case.

Retain the notation of 3.4. Our construction gives a link between $\beta_{t_{2 j-1}-1}^{*}$ (if it is defined) and $\beta_{t_{2 j}-1}^{*}$ which is connected via a sub-chain to the elements in the support of $I_{t_{2 j-1}, t_{2 j}}$ taken in the reverse order. Furthermore the last element in this chain, namely $\beta_{t_{2 j-1}}^{*}$ is linked to $\beta_{t_{2 j}}^{*}$ which is connected via a sub-chain of elements in the support of $I_{t_{2 j}, t_{2 j+1}}$ taken in their natural order to $\beta_{t_{2 j+1}-1}^{*}$, by repeating the pattern in Figure 1 the appropriate number of times. This process is repeated till one reaches $\beta_{t_{2 k-3}-1}^{*}$ which lies just above the last turning point in $A$ with positive signature. Thus $\beta_{t_{2 j-1}-1}^{*}$ is connected via a sub-chain to $\beta_{t_{2 k-3}-1}^{*}$.

A similar (reversed) phenomenon occurs in a region of negative signature. In particular $\beta_{t_{2 k-1}}^{*}$ is connected via a sub-chain to $\beta_{t_{2 \ell-2}-1}^{*}$, which is in turn linked via $3.4(*)$ to $\beta_{t_{2 k-3}-1}^{*}$. This can be illustrated by simply making a mirror reflection of Figure 1 perpendicular to its main axis (in simple language turning it upside down).

If $\iota_{1}$ is not reduced to an isolated value, then $\beta_{t_{2 k-1}}^{*}$ is connected via a sub-chain to $\beta_{t_{2 k-3}}^{*}$ which is in turn linked to $\beta_{t_{2 \ell-2}}^{*}$ via $3.4(* *)$, the latter being connected by a sub-chain to $\beta_{t_{2 \ell-1}-1}^{*}$.

Thus using a line to designate a link or a sub-chain we may summarize the above as

$$
\beta_{t_{2 j-1}-1}^{*}-\beta_{t_{2 k-3}-1}^{*}-\beta_{t_{2 \ell-2}-1}^{*}-\beta_{t_{2 k-1}}^{*}-\beta_{t_{2 k-3}}^{*}-\beta_{t_{2 \ell-2}}^{*}-\beta_{t_{2 \ell-1}-1}^{*}
$$

Except for the two extreme terms the links or sub-chains between these elements are illustrated in the left hand side of Figure 3. 


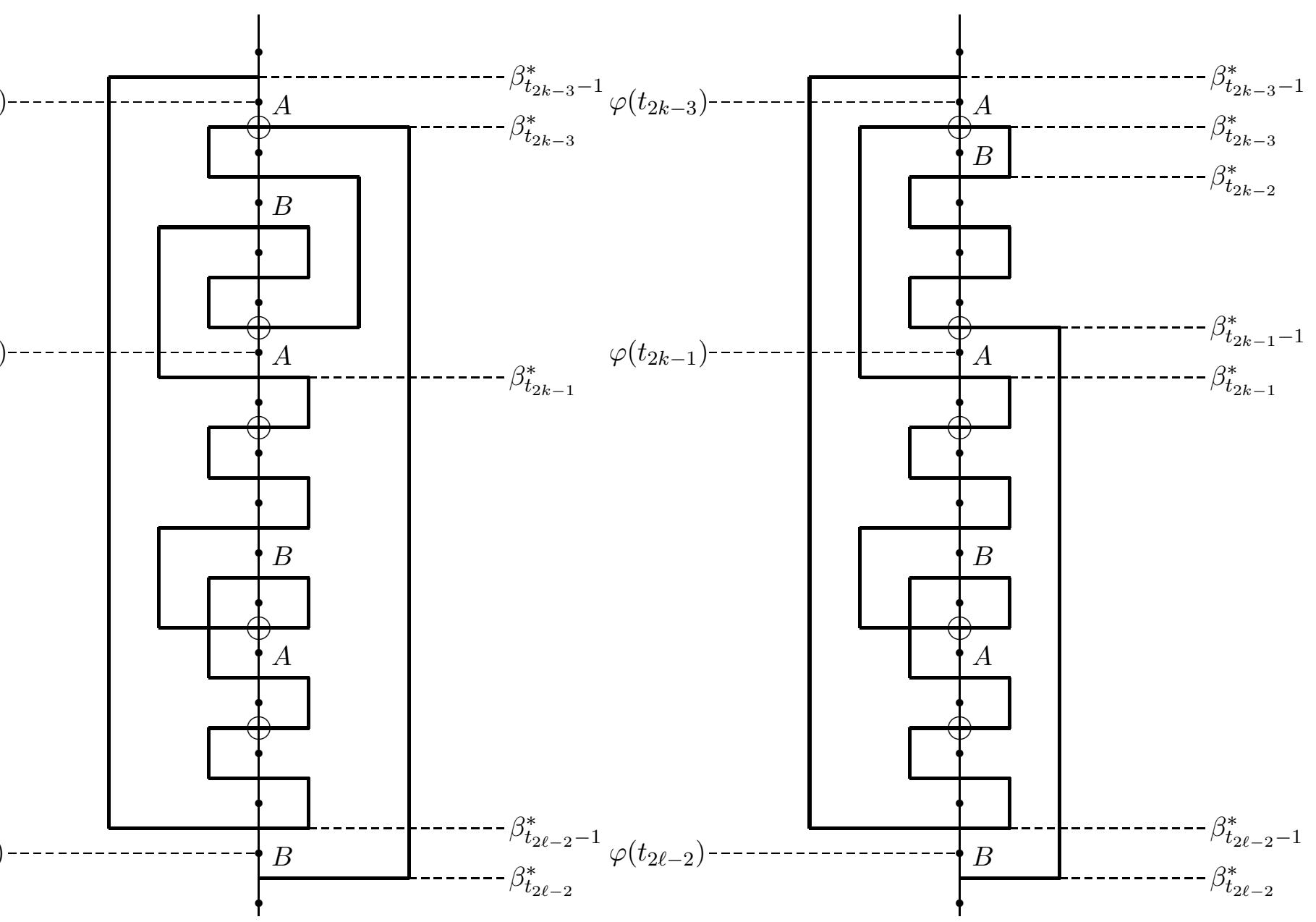

Figure 3.

The left (resp.) right figure illustrates the links or sub-chains in $(*),(* *))$ (resp. $(*),(* *$ $*)$ ) of 3.4. The conventions of Figures 1,2 apply. Compared to Figure 2 one has $\ell=k+2$.

If $\iota_{1}$ is reduced to an isolated value, one checks (taking account of the alternating signs of the $\left.\epsilon_{i}\right)$ that $\beta_{t_{2 \ell-2}}^{*}$ is linked to $\beta_{t_{2 k-1}-1}^{*}$ via $3.4(* * *)$. Moreover the latter is connected by a sub-chain to $\beta_{t_{2 k-2}}^{*}$ which is linked to $\beta_{t_{2 k-3}}^{*}$ in turn linked to $\beta_{t_{2 k-1}}^{*}$. (See Figure 3). In this we remark that $\beta_{t_{2 k-1}}^{\prime}=\beta_{t_{2 k-1}}+\iota_{2}$ and $\beta_{t_{2 k-2}}^{\prime}=\beta_{t_{2 k-2}}+\iota_{1}$.

As before we may summarize the above as

$$
\beta_{t_{2 j-1}-1}^{*}-\beta_{t_{2 k-3}-1}^{*}-\beta_{t_{2 \ell-2}-1}^{*}-\beta_{t_{2 k-1}}^{*}-\beta_{t_{2 k-3}}^{*}-\beta_{t_{2 k-2}}^{*}-\beta_{t_{2 k-1}-1}^{*}-\beta_{t_{2 \ell-2}}^{*}-\beta_{t_{2 \ell-1}-1}^{*} .(* *)
$$


Except for the two extreme terms the links or sub-chains between these elements are illustrated in the right hand side of Figure 3.

In both cases $\beta_{t_{2 j-1}-1}^{*}$ is connected via a sub-chain to $\beta_{t_{2 \ell-1}-1}^{*}$ and the process is then repeated. Notice that in both cases the sub-chain passes through all the $\beta^{*}$ of the left hand or right hand side of the figure.

We may summarize the above by the

Lemma. Condition a) of [2.6 is satisfied by $\Pi^{*}$.

Remark. This result can be read off more easily though less rigorously from Figure 4 which is a paradigm for the general case (except when $\iota_{1}$ is an isolated value; but then one combines it with Figure 3).

3.7. We define a partial order $\leq$ on $I$ as follows. The smallest elements are those $i \in I$ for which $\beta_{i}$ is unchanged. If $\iota_{1}$ as defined in 3.4 is reduced to an isolated value say $\beta_{j-1}$, then we set $\beta_{j}>\beta_{j-1}$ recalling that $\beta_{j-1}$ is left unchanged and $\beta_{j}^{\prime}=\beta_{j}+\beta_{j-1}$.

If $m=1$ in $3.4(* * *)$, then $\beta_{s}$ occurring there is changed by a simple interval value namely $\iota_{3}$. However for the present purposes it is convenient to view this as a compound interval value. Then the largest elements of $i \in I$ are just those for which $\beta_{i}$ is changed by adding a compound interval value, namely when $i=t-1, s$ in the notation of 3.4, within that double signature change. Continuing with this convention we may easily observe that if $\beta_{j}^{\prime}=\beta_{j}+\iota$ is a simple interval value, then the $\beta_{i}$ in the support of $\iota$ are unchanged (see Figure 2, for example). Thus if we let $j \in I$ for which $\beta_{j}$ is changed by a simple interval value, to be the second largest elements of $\mathrm{I}$, it follows that $\leq$ is well-defined and lifts to a total order (which we also denote by $\leq$ ) giving the

Lemma. With respect to $\leq$ the transformation taking the $\epsilon_{i} \beta_{i}$ to $\beta_{i}^{*}$ is triangular with ones on the diagonal.

3.8.

Proposition. $\Pi^{*}$ satisfies conditions $a$ ), b) and d) of [2.6.

Proof. Condition a) is just Lemma 3.6. Condition b) is verified by Lemma 2.8 and the fact that the added interval values are always a sum of an odd number of sequentially adjacent simple interval values.

For condition d) we remark that the $\epsilon_{i} \beta_{i}: i \in I$ are roots and therefore are either positive or negative roots with respect to $\Pi^{*}$. Yet they must be positive roots by Lemma 3.7. 

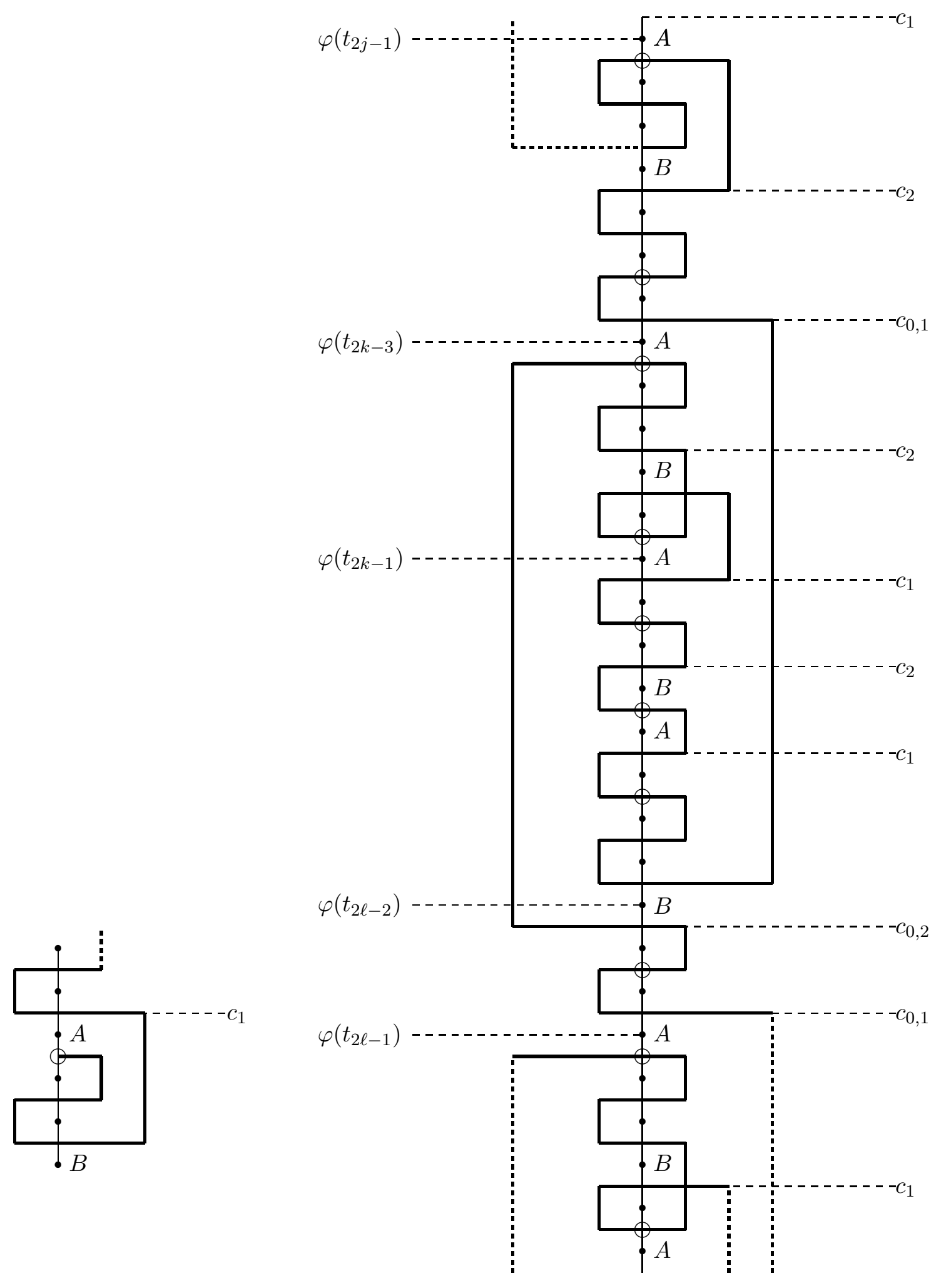
Figure 4.

A diagrammatic presentation of the proof of Lemma 3.6, though of course one could increase the size of the regions of positive and negative signature. The conventions are those of Figures 1,2. The diagram on the left describes what happens if the last element of $B$ is an end point. Again if $\varphi\left(t_{2 \ell-2}\right)$ is replaced by $\varphi(n)=b$, that is to say $\ell$ is not defined, all lines which lie partly or completely below the end point $b$ are removed. If the first element of $A$ is an end point then one omits the dashed lines starting just above the first element of $B$. In this case the lines start at the undecided point, namely $\varphi\left(t_{2}\right)$.

3.9. By Lemma 2.10, condition c) of 2.6 can only fail to hold if $\beta_{e}$ is an unchanged boundary value (that is $\beta_{e}^{\prime}=\beta_{e}$ ) of a unique turning point $\varphi(t) \in B$ which in particular does not have an isolated value as a neighbour. This may be either an internal turning point or an end point. The latter case is essentially a degeneration of the former which we consider first.

The procedure to obtain $\Pi^{*}$ must be modified in the above situation. To be transparent we first recall some features of the description of $\Pi^{*}$ and then define the modified simple root system which we shall denote by $\Pi^{* *}$. Here we recall that $\Pi=\left\{\beta_{i}\right\}_{i \in I}$ is a simple root system of type $A_{n-1}$. In this $\beta_{i-1}, \beta_{i+1}$ will be said to be the neighbours of $\beta_{i}$.

In what follows $\iota_{1}, \iota_{2}$ are interval values but not necessarily those defined in 3.4 .

Let $\beta_{f}$ denote the second boundary value of the internal turning point $\varphi(t)$ defined above. By our assumption and rule 2) of 3.1 , this boundary value must be changed, that is to say we have

$$
\beta_{f}^{\prime}=\beta_{f}+\iota_{1},
$$

where $\iota_{1}$ is an interval value. Moreover this interval value starts at $\beta_{e}$ and so $\iota_{1}-\beta_{e}$ is a root. Since $\beta_{f}$ is a non-nil boundary value, Lemma 2.8 forces it to admit a neighbour $\beta_{f^{\prime}}$ in the simple interval containing $\beta_{f}$. Moreover again by Lemma 2.8 either $\beta_{f^{\prime}}$ is not a boundary value or it is nil and so by rules 1), 2) of 3.1, it is unchanged. That is

$$
\beta_{f^{\prime}}^{\prime}=\beta_{f^{\prime}}
$$

From the definition of $\iota_{1}$ given in 3.4, 3.5, it follows that $\beta_{f^{\prime}}+\beta_{f}+\iota_{1}$ is root.

The fact that

$$
\beta_{e}^{\prime}=\beta_{e}
$$

means that either there is a unique $i(e) \in I$, or simply $i$, such that

$$
\beta_{i}^{\prime}=\beta_{i}+\iota_{2}
$$

for some interval value $\iota_{2}$, with $\beta_{i}^{\prime}-\beta_{e}$ being a root, or $\beta_{e}^{*}$ is at the end point of the Dynkin diagram for $\Pi^{*}$ and in this case we say that $i(e)$ is not defined.

Since $\beta_{e}$ is a non-nil boundary value, Lemma 2.8 forces it to admit a neighbour $\beta_{e^{\prime}}$ in the simple interval containing $\beta_{e}$. The same argument for $\beta_{f^{\prime}}$ given above shows that

$$
\beta_{e^{\prime}}^{\prime}=\beta_{e^{\prime}}
$$

From the definition of $\iota_{2}$ given in 3.4, 3.5, it follows that $\iota_{2}-\beta_{e}$ and $\left(\iota_{2}-\beta_{e}\right)-\beta_{e^{\prime}}$ are roots. 
Since $\beta_{i}$ is a non-nil boundary value to an element of $A$, it admits by Lemma 2.8, a unique neighbour $\beta_{i^{\prime}}$ in the same interval. Moreover $\beta_{i^{\prime}}$ cannot be a nil boundary value (because then it would be a boundary value to an element of $B$ and this would contradict Lemma 2.7(ii)) and it cannot be a non-nil boundary value either (because this would contradict Lemma 2.8). Hence it is not a boundary value and so is unchanged by rule 1) of 3.1, that is

$$
\beta_{i^{\prime}}^{\prime}=\beta_{i^{\prime}}
$$

In particular the fact that $\beta_{i}$ and $\beta_{i^{\prime}}$ are neighbours implies that $\beta_{i}^{\prime}$ and $\beta_{i^{\prime}}^{\prime}$ are neighbours and we designate this as $\beta_{i}^{\prime}=\beta_{i^{\prime}}^{\prime}$, with the sign being that of the scalar product. (For the starred quantities $\beta_{i}^{*}=\epsilon_{i} \beta_{i}$, one recalls that all scalar products have non-positive signs.)

As in [3.6. one checks that $\beta_{e^{\prime}}=\beta_{e^{\prime}}^{\prime}$ is linked via $\iota_{1}$ through a chain defined by nonvanishing scalar products of neighbours to $\beta_{f}^{\prime}=\beta_{f}+\iota_{1}$. We write this as $\beta_{e^{\prime}}^{\prime}=\ldots \pm \beta_{f}^{\prime}$, with the signs having the same meaning as before. Thus (previous to our proposed modification) we obtain the chain

$$
\beta_{i^{\prime}}^{\prime}=\beta_{i}^{\prime} \pm \beta_{e}^{\prime}=\beta_{e^{\prime}}^{\prime}=\ldots \pm \beta_{f}^{\prime}-\beta_{f^{\prime}}^{\prime}
$$

Now we make the following modification (using a double prime to make the distinction clear and writing $\beta_{i}^{* *}:=\epsilon_{i} \beta_{i}^{\prime \prime}$, with $\left.\Pi^{* *}=\left\{\beta_{i}^{* *}\right\}_{i \in I}\right)$. Here exactly three double primed elements are distinct from the single primed elements and only these are described below.

Set

$$
\beta_{i}^{\prime \prime}=\beta_{i}+\iota_{2}-\beta_{e}, \beta_{e}^{\prime \prime}=-\iota_{1}, \beta_{f}^{\prime \prime}=\beta_{f} .
$$

Using the above observations and in particular the linking role of $\iota_{1}$, we obtain the chain

$$
\beta_{i^{\prime}}^{\prime \prime}=\beta_{i}^{\prime \prime} \pm \beta_{e^{\prime}}^{\prime \prime}=\ldots-\beta_{e}^{\prime \prime} \pm \beta_{f}^{\prime \prime}-\beta_{f^{\prime}}^{\prime \prime}
$$

If $i(e)$ is not defined then the first two terms in both $(*)$ and $(* *)$ are absent.

The transition between $(*)$ and $(* *)$ above is illustrated in the passage of the left to the right hand side of Figure 5 (resp. Figure 6) when $\iota_{1}=\iota_{2}$ is a simple (resp. compound) interval value. Observe how the long link between $\beta_{e^{\prime}}^{\prime}$ and $\beta_{f}^{\prime}$, propagated in the first instance through $\iota_{1}$, becomes transformed to a long link between $\beta_{e^{\prime}}^{\prime \prime}$ and $\beta_{e}^{\prime \prime}$ similarly propagated in the first instance through $\iota_{1}$. 


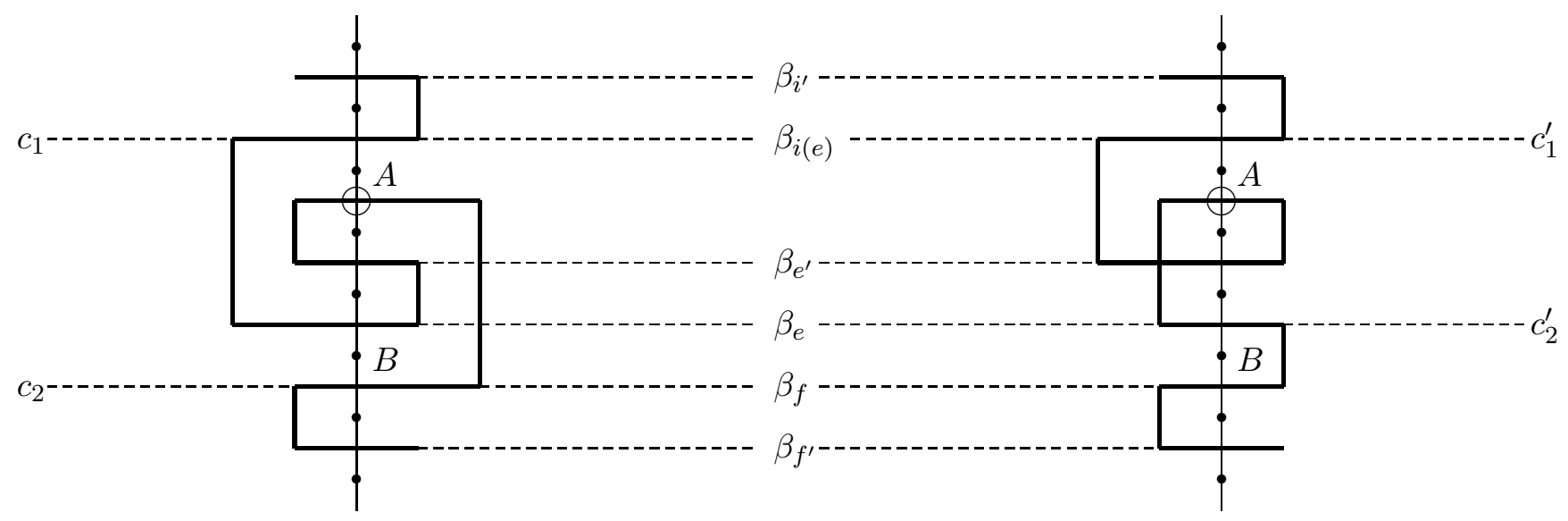

Figure 5.

Passing from left to right illustrates the transition from $(*)$ to $(* *)$ in [3.9, when $\iota_{1}=\iota_{2}$ and is a simple interval value. The conventions of Figure 1 apply. The new changed values according to the discussion following $(*)$ of 3.9 are indicated by a prime. 
$i(e)$ is not defined and we obtain $(*)$ with just the two central terms. Then we set

$$
\beta_{e}^{\prime \prime}=-\iota
$$

and we obtain $(* *)$ with just the two central terms.

Theorem. In the above construction of $\Pi^{* *}$, conditions a)-d), of [2.6 hold.

Proof. Retain the notation of 3.9 ,

Comparison of $(*)$ and $(* *)$ shows that condition a) for $\Pi^{*}$ (verified in Proposition 3.8) implies condition a) for $\Pi^{* *}$.

To show that condition d) holds observe that we still have $\epsilon_{i} \beta_{i}=\beta_{i}^{*}+\sum_{j \in I} \mathbb{N} \beta_{j}^{*}$, for all $i \in I \backslash\{e\}$ and so these elements are positive roots with respect to $\Pi^{*}$.

By contrast $\beta_{e}^{\prime \prime}=-\iota_{1}=-\beta_{e}-\ldots$, and so $\epsilon_{e} \beta_{e}$ does not lie in $\mathbb{N}^{* *}$. However condition d) does not require this.

Thus condition d) holds for $\Pi^{* *}$.

Observe that $\iota_{1}$ expressed as an element of $\pi$ admits a positive (resp. negative) coefficient of $\alpha_{p}$ if $\beta_{e}$ lies above (resp. below) $\varphi(t) \in B$ and that $\epsilon_{e}=1$ (resp. -1). We conclude that condition b) also holds for $\beta_{e}^{* *}$ and hence for $\Pi^{* *}$.

Finally condition c) holds by construction.

3.10. The case when positive and negative need to be interchanged in 3.4, which can occur if $p$ is even, is similar. We sketch the necessary changes below.

Recall the notation of 3.3 and in particular that $j_{1}=1$. Before we had assumed the first of the two possibilities in 3.3 , namely that $\operatorname{sg}\left(j_{1}\right)=1$, to hold. Now we assume the second possibility, namely that $\operatorname{sg}\left(j_{1}\right)=-1$, to hold. Fix a positive odd integer $u \leq r$ and set $j=j_{u}, k=j_{u+1}, \ell=j_{u+2}$.

When only $j$ is defined or when all three are defined the solution we adopt is just the "mirror image" of that described in 3.4. Indeed reading indices in the opposite direction (more precisely applying the involution $i \mapsto n-i$ to $I$ it follows that for example a negative to positive to negative signature change becomes a positive to negative to positive signature change. (Here we do not mean to imply a configuration produced by a coprime pair will transform to a configuration produced by another coprime pair. Indeed our formalism allows for some configurations not necessarily coming from coprime pairs. See 3.11.)

It remains to consider the case of a negative to positive signature change, that is when just $j, k$ above are defined. Surprisingly this is not quite a degeneration of the case when all three are defined.

We remark that to describe a negative to positive signature change repeated more than once (say twice to be specific) then we match a negative to positive to negative signature change with a negative to positive signature change (as illustrated by the right hand side of Figure 7 below).

Set $t=t_{2 k-2}$ and let $s$ be the last turning point which occurs in $A$. One easily checks that the undecided element $d$ is just $\varphi(t)$. We leave $\beta_{t}$ unchanged (which is just what we would do if $\ell$ were defined) and set $\beta_{t-1}^{\prime}=\beta_{t-1}+\iota_{t, s}$. The result is illustrated in Figure 7 . In view of this last change we have labelled the changed element $\beta_{t-1}^{\prime}$ by $c_{0,2}$ in Figure 7 . 


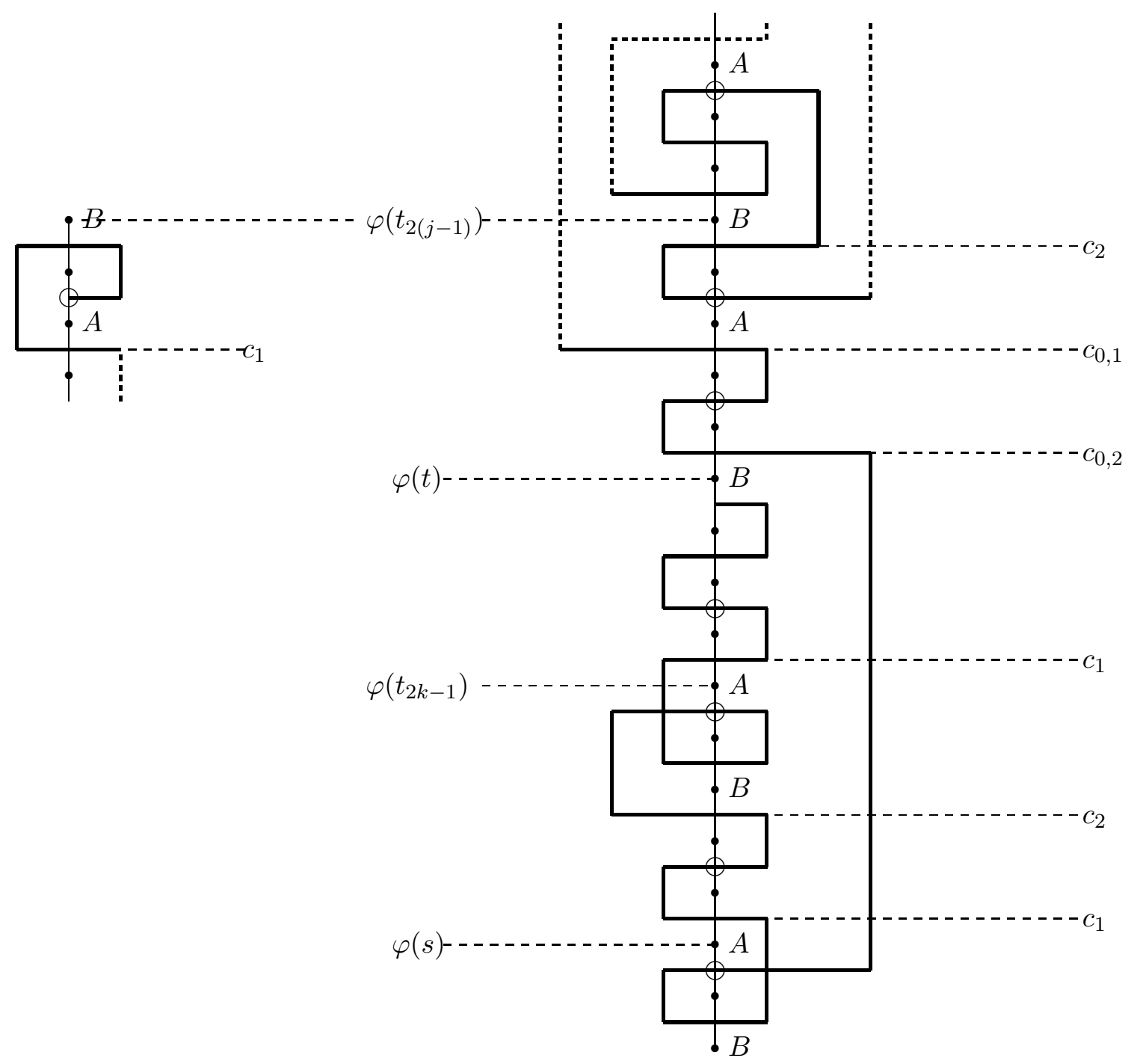

Figure $\%$

This illustrates the negative to positive signature change as discussed and in the notation of [3.10, more precisely in the special case when $j=k-1$. The conventions are those of Figures 1,2. The left hand side describes the situation when $t_{2(j-1)}$ is a starting point. The right hand side describes the situation when $\varphi\left(t_{2 k-1}\right)$ has positive signature but is preceded by a component of negative signature and this being repeated any number of times. Here the top half of the diagram must and does match the negative to positive to negative 
configuration which can be obtained by inverting Figure 4. Curiously the bottom half of the diagram does not match the positive to negative to positive configuration from Figure 4, nor indeed need it do so. An end point of $\Pi^{*}$ in the sense of its Dynkin diagram is $\beta_{t}^{*}$. For the diagram on the left a second end point is $\beta_{t_{2 j-1}-1}^{*}$.

The modification of this construction when the exceptional value is not changed is exactly as in 3.9. The extension of Theorem 3.9 to this case is proved similarly.

3.11. Although the solution we gave to Conditions a)-d) of 2.6 is unambiguous, one can easily check that other solutions can exist for certain coprime pairs $p, q$.

The number of coprime pairs grows at most linearly with $n$ whilst the number of possible signatures grows exponentially. Thus to obtain all possible signatures one would need to have increasingly large gaps between turning points. In particular we do not claim that the particular arrangements described in Figure 4 actually arise from a coprime pair.

3.12. Combining Theorem 3.9 with the remarks in 1.8, 2.6 and 3.10, we obtain the

Corollary. Suggestion 1.4 holds for a (truncated) biparabolic of index 1.

\section{REFERENCES}

[1] N. Bourbaki, Éléments de mathématique. (French) [Elements of mathematics] Groupes et algèbres de Lie. Chapitres 4, 5 et 6. [Lie groups and Lie algebras. Chapters 4, 5 and 6] Masson, Paris, 1981.

[2] J-Y. Charbonnel and A. Moreau, The index of centralizers of elements of reductive Lie algebras. Doc. Math. 15 (2010), 387-421.

[3] J. Dixmier, Algèbres enveloppantes. (French) [Enveloping algebras] Reprint of the 1974 original. Les Grands Classiques Gauthier-Villars. [Gauthier-Villars Great Classics] Éditions Jacques Gabay, Paris, 1996.

[4] F. Fauquant-Millet and A. Joseph, Semi-centre de l'algèbre enveloppante d'une sous-algèbre parabolique d'une algèbre de Lie semi-simple, Annales Scientifiques de l'École Normale Suprieure, Volume 38 (2) (2005), pp. 155-191.

[5] F. Fauquant-Millet and A. Joseph, La somme dans faux degrés - un mystère en théorie des invariants. (French) [The sum of the false degrees - a mystery in the theory of invariants] Adv. Math. 217 (2008), no. 4, 1476-1520.

[6] W. A. de Graaf, Computing with nilpotent orbits in simple Lie algebras of exceptional type. LMS J. Comput. Math. 11 (2008), 280-297.

[7] A. Joseph, On semi-invariants and index for biparabolic (seaweed) algebras. II. J. Algebra 312 (2007), no. 1, 158-193.

[8] A. Joseph, Parabolic actions in type $A$ and their eigenslices. Transform. Groups 12 (2007), no. 3, 515-547.

[9] A. Joseph, A slice theorem for truncated parabolics of index one and the Bezout equation. Bull. Sci. Math. 131 (2007), no. 3, 276-290.

[10] A. Joseph, Invariants and slices for reductive and biparabolic coadjoint actions, Lecture Notes Weizmann 20/2/2007, revised 7/1/2010, available from http://www.wisdom.weizmann.ac.il/ gorelik/agrt.htm

[11] A. Joseph, Slices for biparabolic coadjoint actions in type A, Journal of Algebra, Volume 319 (12) (2008),pp. 5060-5100.

[12] A. Joseph, Compatible adapted pairs and a common slice theorem for some centralizers. Transform. Groups 13 (2008), no. 3-4, 637-669. 
[13] A. Joseph, An Algebraic Slice in the Coadjoint Space of the Borel and the Coxeter Element, preprint, Weizmann 2009.

[14] A. Joseph and D. Shafrir, Polynomiality of invariants, unimodularity and adapted pairs, Transform. Groups (to appear).

[15] B. Kostant, The principal three-dimensional subgroup and the Betti numbers of a complex simple Lie group. Amer. J. Math. 81 (1959), 973-1032.

[16] B. Kostant, Lie group representations on polynomial rings. Amer. J. Math. 85 (1963), 327-404.

[17] A. Ooms and M. Van den Bergh, A degree inequality for Lie algebras with a regular Poisson semi-center, J. Algebra 323 (2010), no. 2, 305-322.

[18] D. Panyushev, A. Premet and O. Yakimova, On symmetric invariants of centralisers in reductive Lie algebras. J. Algebra 313 (2007), no. 1, 343-391.

[19] V. L. Popov, Sections in invariant theory. The Sophus Lie Memorial Conference (Oslo, 1992), 315361, Scand. Univ. Press, Oslo, 1994.

[20] M. Rosenlicht, On quotient varieties and the affine embedding of certain homogeneous spaces. Trans. Amer. Math. Soc. 1011961211223.

[21] O. S. Yakimova, The index of centralizers of elements in classical Lie algebras. (Russian) Funktsional. Anal. i Prilozhen. 40 (2006), no. 1, 52-64, 96; translation in Funct. Anal. Appl. 40 (2006), no. 1, 42-51.

[22] O. S. Yakimova, A counterexample to Premet's and Joseph's conjectures. Bull. Lond. Math. Soc. 39 (2007), no. 5, 749-754. 